\title{
Role of survivin in acute lung injury: epithelial cells of mice and humans
}

\author{
Yasuhiro Terasaki ${ }^{1}$, Mika Terasaki ${ }^{1}$, Hirokazu Urushiyama ${ }^{1}$, Shinya Nagasaka ${ }^{1}$, Mikiko Takahashi ${ }^{1}$, Shinobu Kunugi ${ }^{1}$, \\ Arimi Ishikawa', Kyoko Wakamatsu', Naomi Kuwahara', Koichi Miyake ${ }^{2}$ and Yuh Fukuda
}

Survivin, an inhibitor of apoptosis, regulates cell division and is a potential target for anticancer drugs because many cancers express high survivin levels. However, whether survivin would be toxic to human lung cells and tissues has not been determined. This report clarified the involvement of survivin in acute lung injury. We used immunohistochemical analysis, immunoelectron microscopy, and real-time reverse transcription-quantitative polymerase chain reaction to study survivin expression and localization in injured mouse and human lungs. We also used cultured human lung epithelial cells (BEAS-2B and A549) to study survivin cytoprotection. Nuclei and cytoplasm of epithelial cells in day 3 and day 7 models of bleomycin-injured lung showed survivin-positive results, which is consistent with upregulated survivin mRNA expression. These nuclei also evidenced double positive findings for proliferating cell nuclear antigen and survivin. Day 7 models had similar Smac/DIABLO-positive and survivin-positive cell distributions. The cytoplasm and nuclei of epithelial cells in lesions with diffuse alveolar damage manifested strong survivin-positive findings. Bleomycin stimulation in both epithelial cell lines upregulated expression of survivin and apoptosis-related molecules. Suppression of survivin expression with small interfering RNA rendered human lung epithelial cells susceptible to bleomycin-induced damage, with markedly upregulated activation of caspase-3, caspase-7, poly (ADP-ribose) polymerase, and lactate dehydrogenase activity and an increased number of dead cells compared with mock small interfering RNA-treated cells. Overexpression of survivin via transfection resulted in these epithelial cells being resistant to bleomycin-induced cell damage, with reduced activation of apoptosis-related molecules and lactate dehydrogenase activity and fewer dead cells compared with results for mocktransfected cells. Survivin, acting at the epithelial cell level that depends partly on apoptosis inhibition, is therefore a key mediator of cytoprotection in acute lung injury. Understanding the precise role of survivin in normal lung cells is required for the development of therapeutic survivin.

Laboratory Investigation (2013) 93, 1147-1163; doi:10.1038/labinvest.2013.103; published online 26 August 2013

KEYWORDS: acute lung injury; apoptosis; bleomycin-induced lung injury; cell division; cell survival; cytoprotection; diffuse alveolar damage

Survivin, a member of the inhibitor of apoptosis family, inhibits caspase-mediated cell death by increasing inhibition of caspase through binding the X-linked inhibitor of apoptosis and hepatitis B X-interacting protein, as well as by binding to the proapoptotic factor Smac/DIABLO, which leads to delayed release of Smac/DIABLO in the cytoplasm. ${ }^{1-5}$ Survivin also has an important cell cycle function in as much as it promotes the assembly and stability of microtubules during the $\mathrm{G}_{2} / \mathrm{M}$ phase. ${ }^{6,7}$

Many human tumors express high levels of survivin, and its tissue levels are closely related to tumor progression and a poor prognosis. ${ }^{8}$ Most tissues also clearly express survivin during development, and deletion of the survivin gene is embryonic lethal. ${ }^{9}$ Most normal adult differentiated tissues have very low levels of survivin expression; however, some normal adult cell types, including thymocytes ${ }^{10}$ and basal colonic and gastric mucosal epithelial cells, ${ }^{11}$ demonstrate distinct survivin expression. Survivin expression is elevated in regenerating liver cells after partial hepatectomy ${ }^{12}$ and in microvessels in peri-infarct regions in the mouse brain. ${ }^{13}$ Further, survivin overexpression can protect gastric epithelial cells from ethanol-induced apoptosis, ${ }^{14}$ partly by interfering

\footnotetext{
${ }^{1}$ Department of Analytic Human Pathology and Nippon Medical School, Tokyo, Japan and ${ }^{2}$ Department of Biochemistry and Molecular Biology, Nippon Medical School, Tokyo, Japan

Correspondence: Dr Y Terasaki, MD, PhD, Department of Analytic Human Pathology, Graduate School of Medicine, Nippon Medical School, 1-1-5 Sendagi, Bunkyo-ku, Tokyo 113-8602, Japan.

E-mail: terasaki@nms.ac.jp

Received 2 January 2013; revised 21 July 2013; accepted 22 July 2013
} 
with effector caspases and serving to stabilize mitochondrial activity, ${ }^{15}$ whereas suppressed survivin expression in mice by antisense or transgenic inactivation leads to spontaneous apoptosis and greater sensitivity to Fas and ischemia hypoxia. ${ }^{13,16,17}$ Survivin expression is therefore important during tissue regeneration, and suppression of survivin may contribute to injury.

Acute respiratory distress syndrome is characterized histologically by diffuse alveolar damage (DAD), with findings such as alveolar edema, hyaline membranes composed of sloughed epithelial cell debris and fibrin, and alveolar lining cell hyperplasia. Both apoptosis and cell proliferation are known as very important pathophysiological processes in acute lung injury, especially for cytoprotection and regeneration of lung epithelial cells after injury. ${ }^{18,19}$ We hypothesized that survivin also has an important role in cytoprotection and regeneration in acute lung injury. We studied the expression and localization of survivin via bleomycin-induced lung injury in mice and DAD in humans. We also studied its cytoprotective effect by analyzing bleomycin-induced apoptotic damage in human cultured lung epithelial cells in which we suppressed survivin expression or induced survivin overexpression.

\section{MATERIALS AND METHODS}

\section{Antibodies}

Antibodies purchased for survivin included the following: AF886; surfactant protein-C (SP-C); surfactant protein A; S100A4; F4/80; Smac/DIABLO, activated caspase-3, and activated poly (ADP-ribose) polymerase (PARP); caspase-3, activated caspase-7, caspase-7, and PARP; GAPDH; and proliferating cell nuclear antigen (PCNA).

\section{The Bleomycin-Induced Lung Injury Mouse Model}

Pulmonary fibrosis was induced in 8-week-old male ICR mice (weight 35-40 g; Sankyo Labo Service Corporation, Inc., Tokyo, Japan) by one intratracheal instillation of bleomycin hydrochloride $(25 \mathrm{mg} / \mathrm{kg}$ body weight; Nippon Kayaku Co., Tokyo, Japan). The Animal Care and Use Committee of Nippon Medical School approved the animal protocols.

\section{Patients}

Lung specimens were obtained via autopsies at the Nippon Medical School Hospital during 1992-2011: 10 DAD autopsy cases and eight autopsy control cases. Table 1 provides background information of the patients (Table 1). The study protocol was approved by the Human Ethics Review Committee of Nippon Medical School.

\section{Immunohistochemistry, Immunoelectron Microscopy, Terminal Deoxynucleotidyl Transferase dUTP Nick-End Labeling (TUNEL), and Evaluation for Survivin-Positive Cells}

Paraffin-embedded mouse and human lung sections were evaluated for survivin, SP-C, Smac/DIABLO, and PCNA and were processed for TUNEL for in situ analysis of DNA fragmentation. Ultrathin sections of the Epon-embedded mouse lung that had been incubated with anti-survivin antibody were investigated using transmission electron microscopy. The ratio of the number of bronchial epithelial cells with survivin-positive results in the nucleus or cytoplasm to the total number of cells was determined in a blind manner for mice. The numbers of alveolar epithelial cells with survivin-positive results in the nucleus or the cytoplasm were counted, per high-power field, for lungs from mice, human

Table 1 Clinical background of patients and control subjects

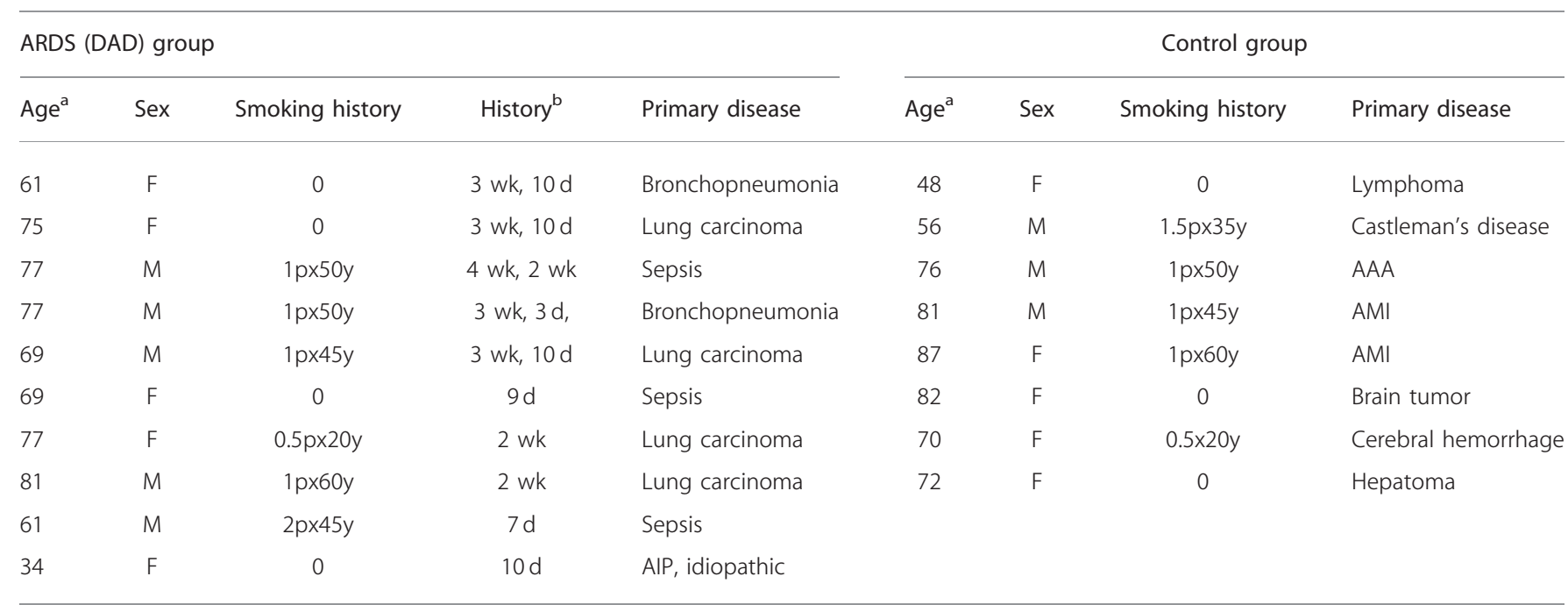

Abbreviations: AAA, abdominal aortic aneurysm; AIP, acute interstitial pneumonia; AMI, acute myocardial infarction; d, day; DAD, diffuse alveolar damage; p, pack/day; wk, week; y, year.

${ }^{\mathrm{a}}$ Mean age in the DAD group was 68.1 years; the male/female ratio $=5: 5$. Mean age in the control group was 71.5 years; the male/female ratio $=5: 3$.

bHistory indicates the duration of clinical events in ARDS. 
DAD cases, and control subjects. The online Supplementary Information provides details of these methods.

\section{Double Labeling for Immunohistochemical and TUNEL Staining}

After endogenous peroxidase activity and nonspecific binding sites were blocked, slides were incubated overnight with the first primary antibody against survivin, followed by incubation with HRP-conjugated anti-rabbit antibodies (Histofine Simple Stain MAX PO (R); Nichirei Biosciences Inc.) and visualization. The primary antibody was inactivated by heat treatment (boiling) to prevent mixed labeling and cross-reaction. ${ }^{20}$ The second primary antibodies against SP-C and S100A4 were incubated overnight, followed by incubation with an alkaline phosphatase (AP)-conjugated anti-goat antibody (Histofine Simple Stain AP (G); Nichirei Biosciences Inc.) and visualization.

After endogenous peroxidase activity and nonspecific binding sites were blocked, slides were incubated overnight with the first primary antibody against PCNA, followed by incubation with HRP-conjugated anti-mouse antibodies (Mousestain Kit PO (M); Nichirei Biosciences Inc.) and visualization. The first primary antibody was inactivated by boiling to prevent mixed labeling and cross-reaction. The second primary antibody against survivin was incubated overnight, followed by incubation with an AP-conjugated anti-rabbit antibody (Histofine Simple Stain AP (R); Nichirei Biosciences Inc.) and visualization.

After TUNEL staining without Mayer's hematoxylin counterstaining, immunohistochemical analysis for suvivin was performed with the incubation of survivin antibody, followed by incubation with HRP-conjugated anti-rabbit antibodies (Histofine Simple Stain MAX PO (R); Nichirei Biosciences Inc.) and visualization.

\section{Real-Time Reverse Transcription-Quantitative Polymerase Chain Reaction (RT-qPCR) Amplification}

Survivin mRNA expression was analyzed by performing realtime PCR. Total RNA was extracted with TRIzol reagent (Qiagen, GmbH, Hilden, Germany) according to the manufacturer's instructions. Ready-to-use primer and probe sets from Applied Biosystems, Foster City, CA, USA (Assay-on-Demand Gene Expression Catalog number Mm00599749_m1, and GAPDH) were used to detect mouse survivin mRNA. Primer and probe concentrations for each target gene were optimized according to the manufacturer's procedure. PCR $\left(2 \mathrm{~min}\right.$ at $50{ }^{\circ} \mathrm{C}, 10 \mathrm{~min}$ at $95^{\circ} \mathrm{C}$, and 45 cycles of $15 \mathrm{~s}$ of denaturation at $95^{\circ} \mathrm{C}$ and $60 \mathrm{~s}$ of annealing at $60^{\circ} \mathrm{C}$ ) was performed on the ABI Prism 7000 Sequence Detection System (Applied Biosystems) with the fluorescent TaqMan methodology. For all experiments, survivin mRNA and GAPDH mRNA were quantified in triplicate, and survivin mRNA was normalized against GAPDH mRNA. Results are expressed relative to the standard sample (SS) $(1 \times \mathrm{SS}=1.0)$.

\section{Cell Culture}

BEAS-2B human bronchial epithelial cells were obtained from American Type Culture Collection (Manassas, VA, USA) and were grown in culture dishes or culture slides coated with type VI collagen (collagen from human placenta; Sigma-Aldrich, Inc., St Louis, MO, USA) with bronchial epithelial cell basal medium as the maintenance medium (Lonza Walkersville, Inc., Walkersville, MD, USA) and with growth supplements (Lonza Walkersville, Inc.) in a $5 \% \mathrm{CO}_{2}$ humidified chamber. Human lung alveolar epithelial A549 cells were obtained from the Cell Research Center for Biomedical Research (Institute of Development, Aging and Cancer, Tohoku University) and were grown in culture dishes or culture slides with maintenance medium RPMI (Life Technologies Corporation, Carlsbad, CA, USA) containing $10 \%$ fetal bovine serum and $1 \%$ penicillin-streptomycin (Life Technologies Corporation) in a $5 \% \mathrm{CO}_{2}$ humidified chamber.

\section{Western Blotting}

For each experiment, western blotting was performed according to the standard procedure. After stripped blots were reacted with the appropriate antibodies, western blotting was performed, and proteins were quantified, with results expressed as ratios to the amount of GAPDH protein.

\section{Detection of Cell Damage}

Culture medium, under indicated conditions, was analyzed for lactate dehydrogenase (LDH) release (signifying cell damage) using Cytotoxicity Detection KitPLUS (Roche Applied Science, Mannheim, Germany) according to the manufacturer's instructions. Optical density (OD) was measured at $490 \mathrm{~nm}$ with a Model 550 Microplate Reader (Bio-Rad Laboratories Inc., Hercules, CA, USA), and five OD results were reported relative to corresponding results for controls (no treatment) in five experiments (control treatment $=1.0$ ). We also assessed cell survival by manually counting cells that were double stained with Annexin V-FITC (BD Biosciences, San Jose, CA, USA; apoptotic cell membranes: green) and $1 \mathrm{mM}$ propidium iodide (PI) (Invitrogen; dead cell nuclei: pink) as well as $5 \mathrm{mM}$ Hoechst 33342 dye (Invitrogen; nuclei of dead and living cells: blue).

\section{Small Interfering RNA (siRNA) Experiments}

siRNA targeting human survivin and nontargeting control siRNA were obtained from Sigma-Aldrich (MISSION siRNA and MISSION siRNA Universal Negative Control, respectively). Transfection was performed with Lipofectamine RNAiMAX reagent (Invitrogen), according to the manufacturer's protocol, in $1 \mathrm{ml}$ of Opti-MEM I-reduced serum medium (Invitrogen).

\section{Survivin Overexpression Studies}

pcDNA3HA-survivin, a survivin expression vector with an N-HA tag, purchased from $\mathrm{CH} 3$ BioSystems, LLC 
(Amherst, NY, USA), was used for survivin overexpression studies, with Lipofectamine 2000 (Invitrogen) and FuGENE HD transfection reagent (Roche Applied Science) used for transfection according to the manufacturer's instructions. Transfection efficiency was controlled by immunoblotting.

\section{Statistical Analysis}

For each data set, arithmetic means and s.e.m values were calculated; Student's $t$-test was used to compare paired or independent variables. One-way ANOVA was used to determine statistical differences among groups. ${ }^{\star} P<0.05$ and ${ }^{*} P<0.01$ were considered statistically significant.

\section{RESULTS}

\section{Expression of Survivin in Acute Lung Injury In Vivo}

To clarify the role of survivin in acute lung injury, we used immunohistochemistry to study survivin expression and localization in bleomycin-injured lungs of mice. We detected slight survivin reactivity in the cytoplasm of bronchial epithelial cells of control adult lungs (Figures 1a and b, arrows). In contrast to control lungs before induction, obvious survivin-positive findings were seen mainly in the nuclei of bronchial epithelial cells and cells of alveolar areas in day 3 bleomycin-injured lung (Figures $1 \mathrm{c}$ and d, arrowheads). Also, day 7 models showed clear positive findings in both the nuclei (arrowheads) and the cytoplasm (arrows) (Figures 1e and f) of bronchial and mainly alveolar epithelial cells. Figure 2 shows many single- and double (arrows)-positive findings for survivin and SP-C (a marker of type II pneumocytes) (Figures 2a-c, serial sections; g and h, serial sections), some single- and double (arrows)-positive results for survivin and S1004A (a marker of fibroblasts) (Figures 2d-f), and some single-positive staining for survivin and F4/80 (a marker of macrophages) (Figures $2 \mathrm{~h}$ and $\mathrm{i}$, arrowheads) with serial sections from day 7 mice. In the day 14 models, survivin-positive results were found mainly in the cytoplasm of epithelial cells, although the survivin-positive findings of the cells tended to be weak as brown color intensity (Figures $1 \mathrm{~g}$ and $\mathrm{h}$, arrows). By immunoelectron microscopic analysis, intracytoplasmic granules with mitochondria-like structures of epithelial cells were positive for survivin in the bleomycin-injured lung of the day 14 model (Figure $2 \mathrm{j}$, arrows).

We semiquantified survivin expression in alveolar and bronchial epithelial cells of bleomycin-injured lung from each group $(n=4)$ by counting, in blind manner, the number of cells positive for survivin in the nucleus or the cytoplasm. We found that survivin expression in the nuclei was upregulated in the day 3 model and showed a peak in the day 7 model and then a reduction in the day 14 model of bleomycin-injured lung (Figures $3 \mathrm{a}$ and $\mathrm{b}$ ). We also found that survivin expression in the cytoplasm of bronchial epithelial cells was upregulated in the day 3 model and peaked in the day 7 model (Figure 3c). The number of cytoplasmic survivin-positive alveolar cells did not show a clear peak in the day 7 model and a reduction in the day 14 model, although the survivin-positive intensity themselves in the day 14 model tended to be weak (Figure 3d). We also quantified survivin mRNA expression in the bleomycin-injured lung by using real-time RT-qPCR. Consistent with the findings obtained for immunostaining, survivin mRNA expression was significantly upregulated in the day 7 models in the bleomycin-injured lung compared with control lung, and expression levels tended to be reduced in the day 14 models (Figure 3e).

As survivin regulates both cell division and cell survival through apoptosis, we studied the expression of survivin and related molecules in bleomycin-injured lungs. The nuclei of some bleomycin-injured epithelial cells showed positive results for survivin with PCNA (DNA synthesis marker) (Figures $4 \mathrm{a}$ and $\mathrm{b}$, arrowheads; Figures $4 \mathrm{~g}-\mathrm{i}$, arrowheads) in serial sections of lungs from the day 7 models. Cells that were positive for survivin and Smac/DIABLO (a proapoptotic factor) had similar distributions in serial sections of epithelial cells (Figures 4c and d, arrows). We did not detect any clear survivin-positive cells with TUNEL-positive staining (Figures $4 \mathrm{e}$ and $\mathrm{f}$, arrows; Figures $4 \mathrm{j}-\mathrm{l}$, arrowheads) in serial sections of lungs from the bleomycin-injured day 7 models. No nonspecific IgG reaction was seen (data not shown).

We also used immunohistochemistry to study survivin expression and localization in human control lungs and DAD lung lesions. In contrast to the slight survivin reactivity in the cytoplasm of bronchial epithelial cells of controls (Figure 5a and b, arrow), strong survivin-positive findings were seen mainly in the cytoplasm and/or nucleus of epithelial cells in DAD lung lesions (Figures $5 \mathrm{c}$ and $\mathrm{d}$, arrows and arrowhead). We saw many positive results for survivin and surfactant protein A (a marker of type II pneumocytes) (Figures $5 \mathrm{~d}$ and $\mathrm{e}$, black arrows) and some positive findings for survivin and CD68 (a marker of macrophages) (Figures $5 \mathrm{~d}-\mathrm{f}$, red arrows) with serial sections. We semiquantified survivin expression in the human alveolar epithelial cells by counting, in blind fashion, the number of survivin-positive cells in the nucleus or cytoplasm and observed upregulated survivin expression in DAD lungs compared with control lungs (Figure 5g).

These results, together with the evidence of upregulated expression of survivin and activation of survivin-related molecules in injured lungs, suggested that survivin in epithelial cells is involved in the pathophysiology of acute lung injury, not only in cancerous tissue cells, in mice and humans.

\section{Expression of Survivin in Lung Epithelial Cells after Bleomycin Treatment In Vitro}

To investigate the role of survivin in lung epithelial cell damage in vitro (as we did in vivo), we evaluated survivin expression in BEAS-2B cells and A549 cells, well-characterized cultured human bronchial epithelial and alveolar epithelial cell lines, respectively, after bleomycin-induced cell 


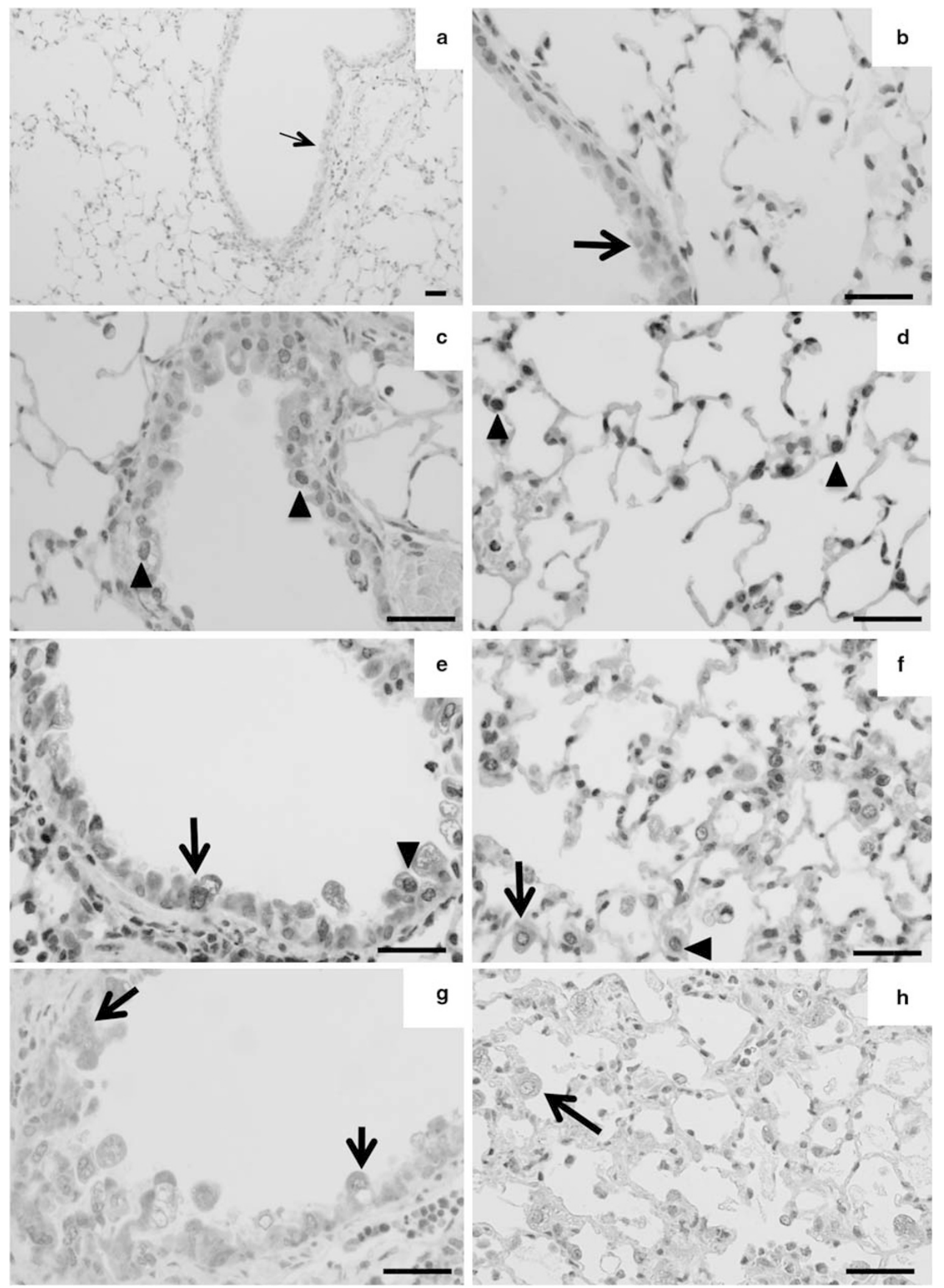

Figure 1 Expression of survivin in bleomycin-injured lungs of mice. Paraffin-embedded lung sections were stained for survivin. (a-c, e, $\mathbf{g}$ ) Bronchial wall. (d, f, h) Alveolar areas. (a and b) Day 0 (controls). (c and d) Day 3. (e and f) Day 7. (g and h) Day 14. Arrowheads and arrows indicate positive findings in the nuclei and cytoplasm, respectively. Scale bar: $50 \mu \mathrm{m}$. 

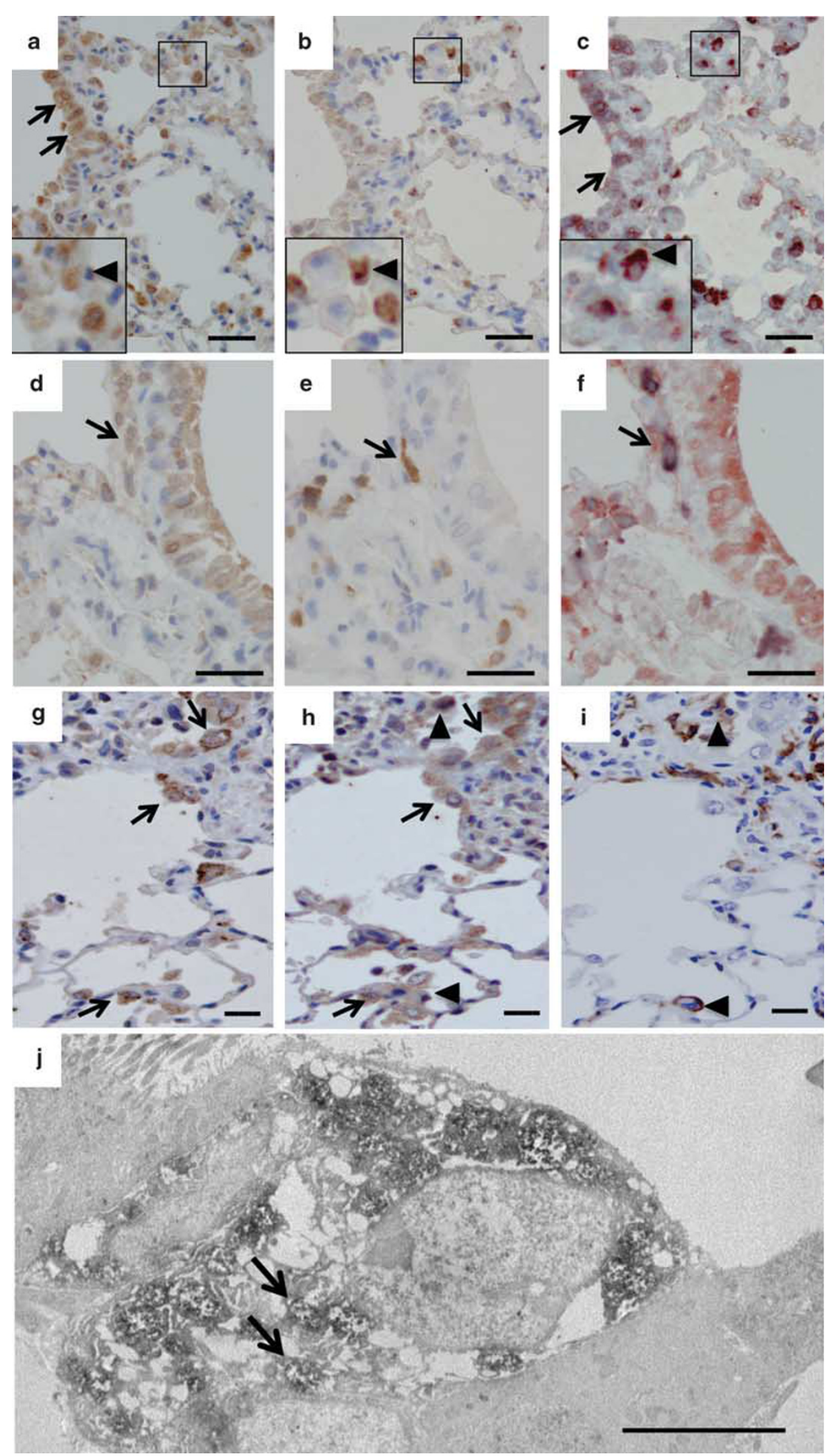
injury. As survivin regulates cell survival in part by antiapoptotic signaling pathways, we investigated the activation of caspase-3, caspase-7, and PARP in addition to evaluating survivin expression. After incubation with a low concentration of bleomycin, BEAS-2B cells demonstrated gradually
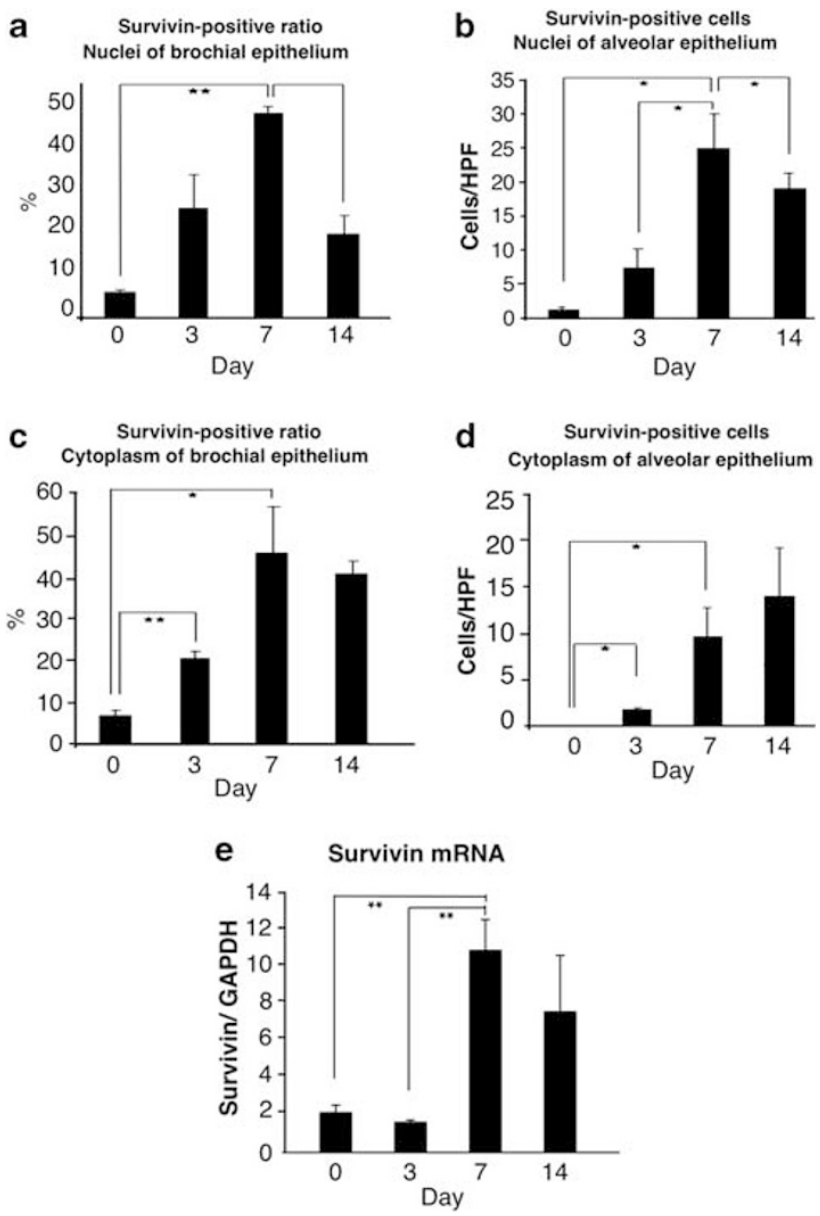

Figure 3 Quantification of survivin in bleomycin-injured lungs of mice. (a-c) Ratios of the number of survivin-positive (nucleus, a; cytoplasm, c) bronchial epithelial cells to the total number of cells were determined for mice in each group-day 0 (control) and days 3, 7, and $14(n=4)$. (b-d) The numbers of survivin-positive (nucleus, $\mathbf{b}$; cytoplasm, $\mathbf{d}$ ) alveolar epithelial cells, per high-power field (HPF), were counted for mice in each group $(n=4)$. (e) Survivin mRNA/GAPDH mRNA ratios for bleomycininjured mouse lung were analyzed by using real-time reverse transcription-quantitative polymerase chain reaction $(n=5)$. Each bar represents the level of survivin mRNA normalized to the level of GAPDH mRNA, shown as a percentage of the control value (day 0 ). ${ }^{*} P<0.05$, ${ }^{* *} P<0.01$. upregulated survivin immunoreactivity, in a time-dependent manner, for up to $12 \mathrm{~h}$, with similar upregulated activated caspase-3, activated caspase-7, and activated PARP (Figures 6a-e). To explore one consequence of the activation of caspase-3, caspase-7, and PARP, we measured the number of dead cells and LDH activity (OD) after bleomycin treatment. Consistent with upregulated activation of caspase-3, caspase7, and PARP, the number of dead cells (PI-positive cells) (Figures $7 \mathrm{a}$ and $\mathrm{b}$ ) and $\mathrm{LDH}$ activity (Figure $7 \mathrm{c}$ ) were also upregulated. Incubation of BEAS-2B cells and A549 cells with a high concentration of bleomycin produced strong immunoreactivity for survivin, which was significantly upregulated at $3 \mathrm{~h}$, with immunoreactivity levels being reduced at $24 \mathrm{~h}$ (Figures $6 \mathrm{f}$ and g; Figures $8 \mathrm{a}$ and b). Activated caspase-3, activated caspase-7, and activated PARP were also upregulated at 3,7 , and $3 \mathrm{~h}$, respectively, in BEAS-2B cells (Figures $6 f, h-j)$, and activated caspase- 3 and activated PARP were upregulated at 3 and $12 \mathrm{~h}$, respectively, in A549 cells (Figures $8 \mathrm{a}-\mathrm{c}, \mathrm{d})$ with upregulation of the number of dead cells (Figures $7 \mathrm{~d}$ and e; Figures $8 \mathrm{e}$ and $\mathrm{f}$ ) and LDH activity (Figure 7f and Figure 8g). Thus, levels of susceptibility (sensitivity) to bleomycin damage differed in the two cell lines, with survivin being upregulated in bleomycin-damaged lung epithelial cells in vitro, just as it was in vivo. These results are consistent with our hypothesis that survivin may have an important role in bleomycin-induced lung epithelial cell damage via apoptotic signaling pathways in vitro and in vivo.

\section{Effect of Survivin Suppression on Bleomycin-Induced Damage of Lung Epithelial Cells}

To investigate the cytoprotective role of survivin in bleomycin-induced lung damage, we incubated BEAS-2B cells and A549 cells with siRNA to suppress survivin expression and analyzed the effect by western blotting with markers for apoptotic signaling pathways, ie, activated caspase-3, activated caspase-7, and activated PARP. Suppression of survivin expression in these cells induced markedly upregulated activation of caspase-3, caspase-7, and PARP compared with mock siRNA-treated BEAS-2B cells (Figures 9a-e) and A549 cells (Figures 10a-c). Consistent with the increased activation of caspase-3, caspase-7, and PARP after survivin suppression, cultured BEAS-2B cells (Figures 9f and g) and A549 cells (Figures 10g-i) demonstrated more severe damage after this treatment, as evidenced by a significantly greater number of apoptotic or dead cells (Annexin V- or PI-positive cells) compared with mock siRNA-treated cells. BEAS-2B cells also

Figure 2 Expression of survivin and cell markers in bleomycin-injured lungs of mice. Paraffin-embedded lung sections from day 7 model mice were stained for survivin (a) and surfactant protein-C (SP-C) (b) and were double stained for survivin/SP-C (c); or were stained for survivin (d) and S1004A (e) and were double stained for survivin/S1004A (f); or were stained for SP-C (g), survivin (h), and F4/80 (i) with each set of serial sections. (a, b; d, e) Mirror images showing co-localization of staining. (a-c, $\mathbf{g}-\mathbf{I})$ Two sets of serial sections, respectively, for cells in the alveoli; (d-f) serial sections for stromal cells. Scale bar: $30 \mu \mathrm{m}$. (j) Immunoelectron microscopic image showing survivin-positive intracytoplasmic granules with mitochondria-like structures (arrows) in Clara cells in the day 14 model of the bleomycin-injured lung. Insets show high-magnification views of areas in rectangles. Scale bar: $5 \mu \mathrm{m}$. 
demonstrated significantly increased LDH activity compared with mock siRNA-treated cells (Figure 9h).

\section{Effect of Survivin Overexpression on Bleomycin-Induced Damage of Lung Epithelial Cells}

To further investigate the cytoprotective role of survivin in bleomycin-induced damage of lung epithelial cells, BEAS-2B and A549 cells were transfected to produce survivin overexpression, and the effect was analyzed by western blotting. Survivin overexpression markedly reduced activation of caspase- 3 , caspase-7, and PARP in both cell lines compared with mock transfection (Figures 10d-f; Figures 11a-e). Consistent with the reduced activation of caspase-3, caspase-7, and PARP after survivin overexpression, cultured A549 cells
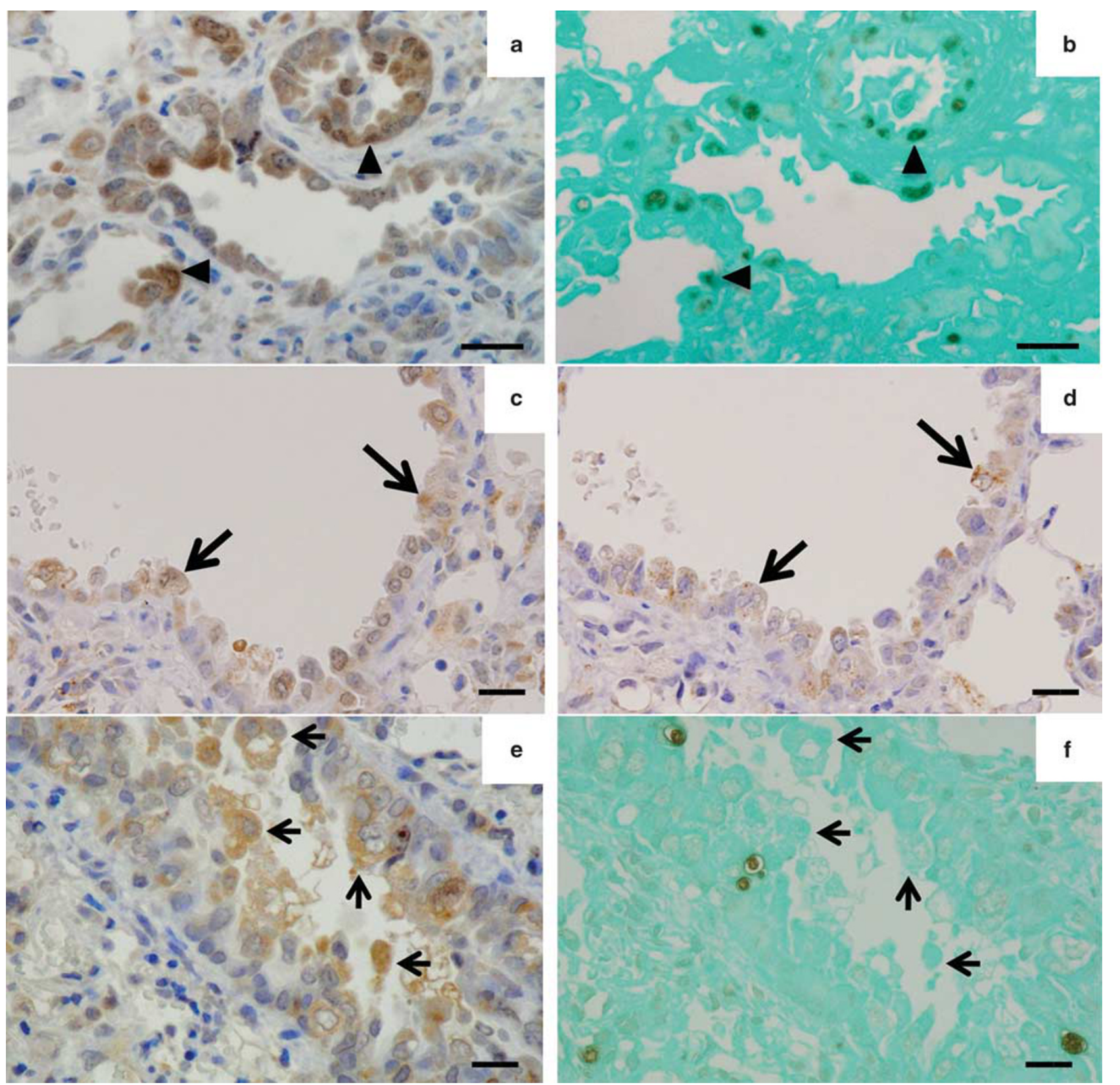

Figure 4 Expression of survivin and related molecules in bleomycin-injured lungs of mice. (a-f) Paired serial paraffin-embedded lung sections from day 7 model mice were stained for survivin (a, c, e), proliferating cell nuclear antigen (PCNA) (b), Smac/DIABLO (d), or terminal deoxynucleotidyl transferase dUTP nick-end labeling (TUNEL) (f). (a, b) Arrowheads indicate survivin- and PCNA-positive nuclei of epithelial cells, respectively. (c, d) Arrows indicate survivin- and Smac/DIABLO-positive cytoplasm of epithelial cells, respectively. (e, f) Arrows indicate survivin-positive and TUNEL-negative cells, respectively. (g-l) Serial paraffin-embedded lung sections from day 7 model mice were stained for PCNA (g), survivin (h-k), or TUNEL (j) or were double stained for survivin/PCNA (i) or survivin/TUNEL (I). (g-i) Arrowheads indicate PCNA-positive (g) and survivin-positive (h) and survivin/PCNA doublepositive (i) nuclei of epithelial cells. (j-I) Arrowheads indicate TUNEL-positive (j) and survivin-negative (k) and TUNEL-positive/survivin-negative (I) epithelial cells. Scale bar: $30 \mu \mathrm{m}$. 

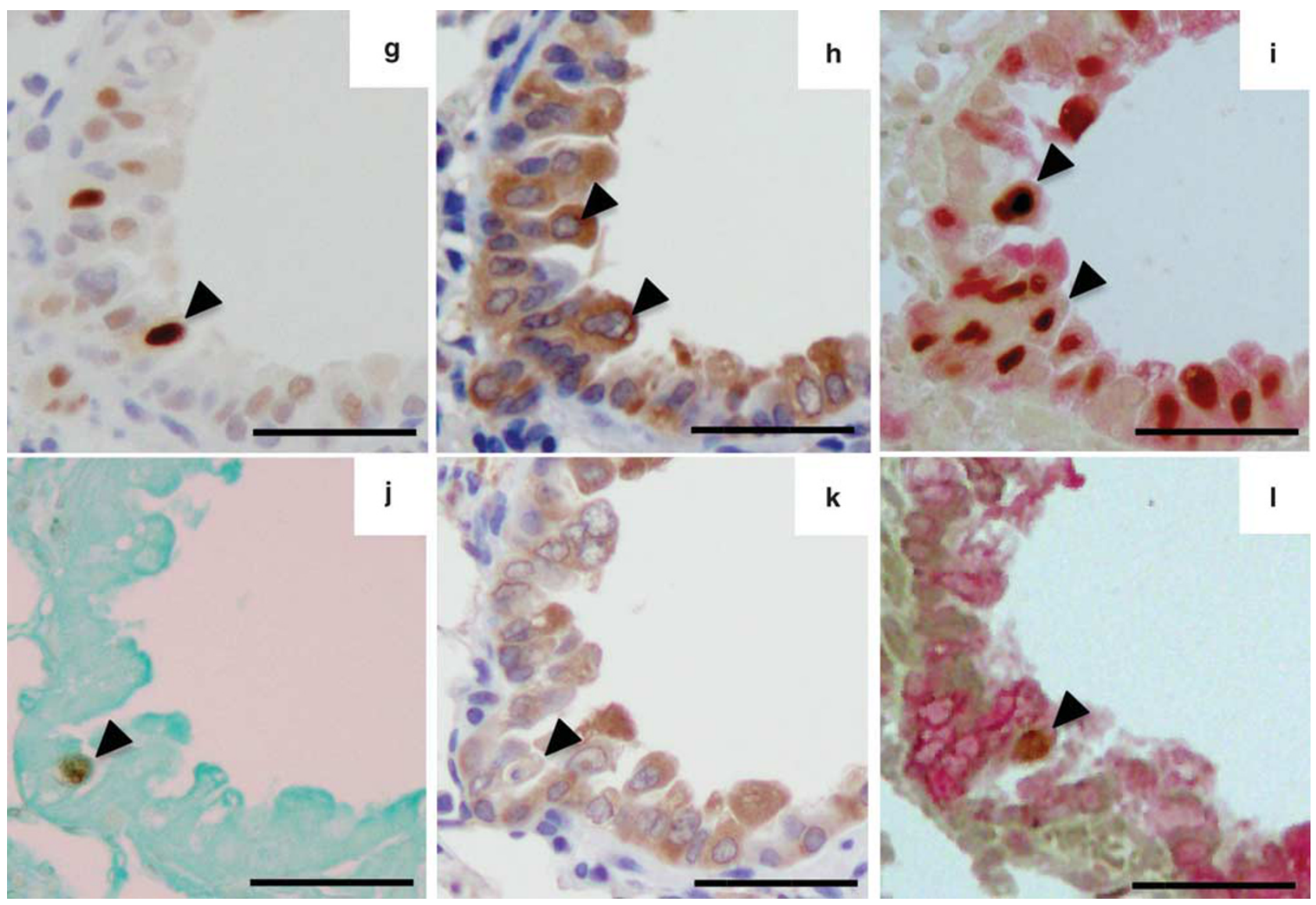

Figure 4 (Continued).

(Figures 10h-j) and BEAS-2B (Figures 11f and g) demonstrated less severe damage after this treatment, as evidenced by a significantly lower number of apoptotic or dead cells (Annexin V- or PI-positive cells) compared with controls. BEAS-2B cells also showed significantly decreased LDH activity (Figure $11 \mathrm{~h}$ ) compared with mock-transfected cells. These results indicate that survivin has a cytoprotective role in epithelial cells in acute lung injury, with the mechanism being apoptosis inhibition.

All these data therefore show that survivin is a critical mediator of cytoprotection in acute lung injury and that it acts at the epithelial cell level, in part, by a mechanism that depends on the inhibition of apoptosis.

\section{DISCUSSION}

This report is the first to clarify the involvement of survivin in acute lung injury. Here, we describe the upregulation of survivin mRNA in bleomycin-induced lung injury in mice. We found clear evidence of the upregulated presence of survivin, with related molecules such as PCNA and Smac/ DIABLO, in epithelial cells of injured lung lesions in mice and in epithelial cells in human cases of DAD. We also showed, by utilizing bleomycin-induced apoptotic damage plus survivin suppression or overexpression, the cytopro- tective effects of survivin on cultured lung epithelial cells. These results thus indicate that survivin may be an important mediator of cytoprotection, not only of malignant cells but also of noncancerous epithelial cells in acute lung injury in mice and humans.

In one report, survivin expression was nearly absent in adult differentiated tissue including the lung. ${ }^{21}$ However, we detected, by RT-qPCR, survivin mRNA in control adult mouse lungs (Figure 3e), and we found slight reactivity for survivin in the cytoplasm of bronchial epithelial cells from control adult mouse lungs and control human lungs (Figures 1a and $\mathrm{b}$; Figures $5 \mathrm{a}$ and $\mathrm{b}$ ). These findings are consistent with a report that identified, by RT-PCR, the survivin gene transcript in $12 \%$ of paired histopathologically normal human lung samples. ${ }^{22,23}$ Endothelial cells, neutrophils, lymphocytes, and mononuclear cells (as well as epithelial cells) may be responsible for survivin mRNA expression seen in control adult mouse lungs. However, we found no notable accumulation of neutrophils, lymphocytes, or mononuclear cells in control mouse lungs. Moreover, survivin expression in endothelial cells was reportedly much lower than that in epithelial cells under physiological conditions. ${ }^{24}$ Thus, bronchial epithelial cells may be the main source of survivin mRNA seen in control lung samples (Figure 3e), results that 

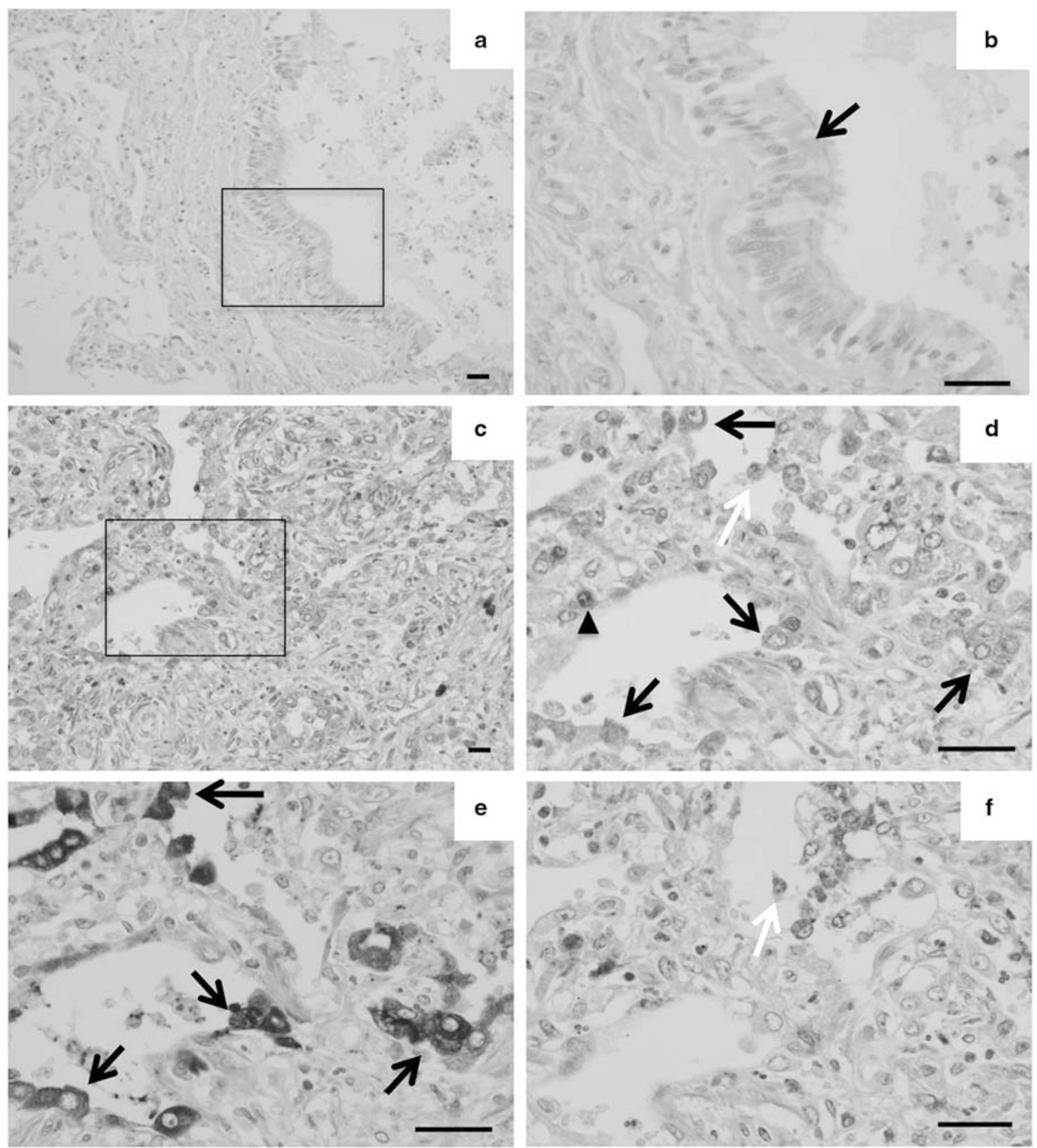

g Survivin-positive

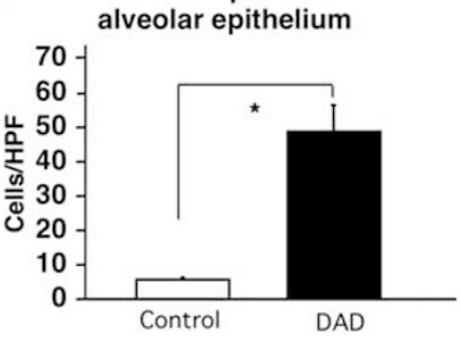

Figure 5 Expression of survivin in human injured lung. $(\mathbf{a}, \mathbf{b})$ Paraffin-embedded lung sections from control cases were stained for survivin. Serial paraffin-embedded lung sections from diffuse alveolar damage (DAD) cases were stained for survivin (c and d), surfactant protein A (e), and CD68 (f). Panels $\mathbf{b}$ and $\mathbf{d}$ show high-magnification views of areas in the rectangles in panels $\mathbf{a}$ and $\mathbf{c}$. Arrowheads and arrows indicate positive findings in the nuclei and cytoplasm, respectively. Scale bar: $50 \mu \mathrm{m}$. (g) The numbers of survivin-positive alveolar epithelial cells, per high-power field, were counted for controls and DAD cases. ${ }^{*} P<0.05$. 

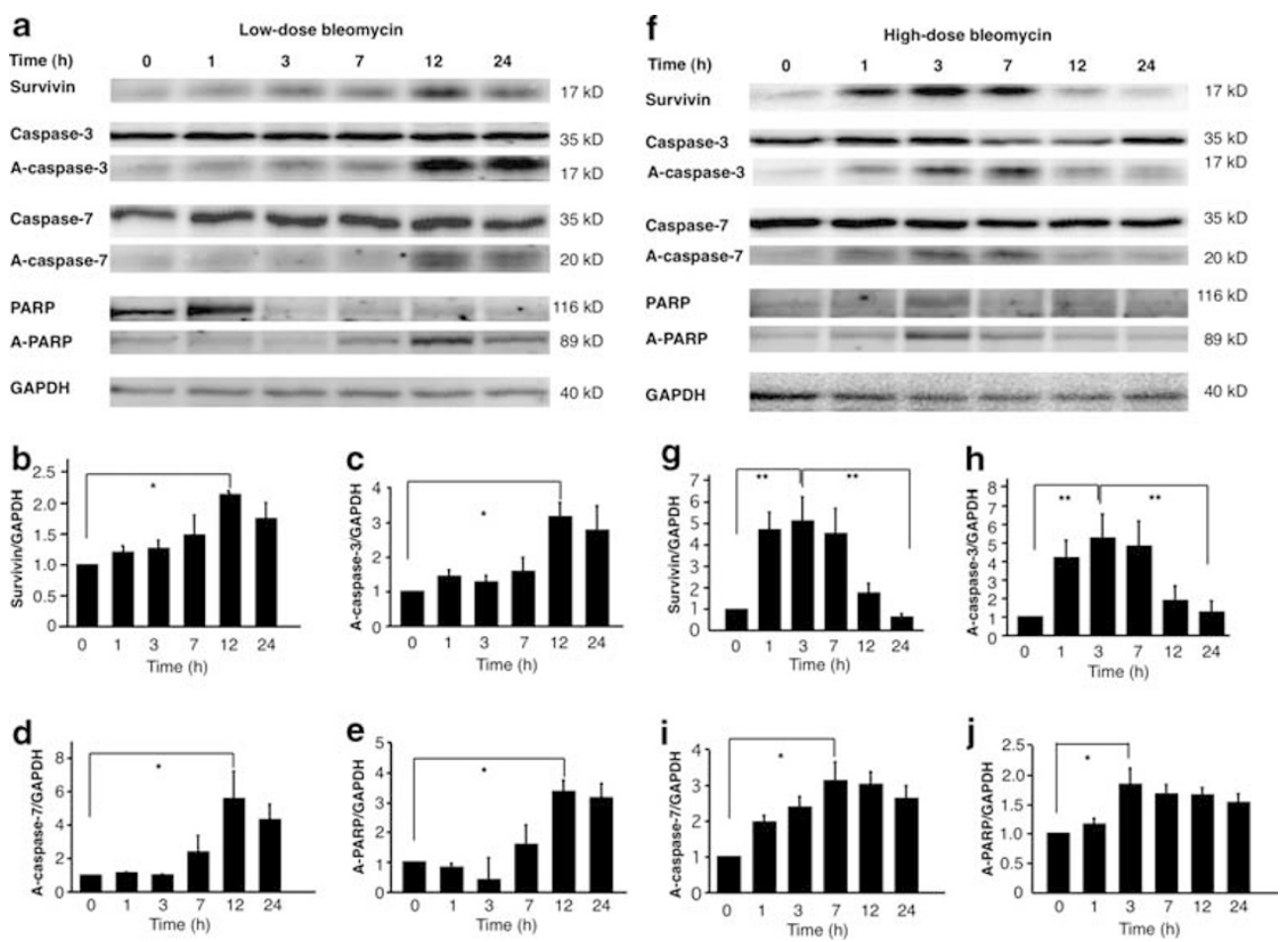

Figure 6 Expression of survivin in human bronchial cells after bleomycin treatment. (a-f) Cell extracts, at indicated times after treatment with bleomycin (low dose or high dose), were subjected to sodium dodecyl sulfate-polyacrylamide gel electrophoresis and were immunoblotted with antibodies against survivin, caspase-3, activated caspase-3 (A-caspase-3), caspase-7, activated caspase-7 (A-caspase-7), poly (ADP-ribose) polymerase (PARP), activated PARP (A-PARP), and GAPDH (control). (b-e, $\mathbf{g - j}$ ) In five experiments similar to that whose results are shown in panels a and $\mathbf{f}$, the amounts of each protein were quantified by means of densitometry and were expressed relative to the amount of GAPDH in those samples. Results are reported relative to those of five controls in five experiments (no treatment $=1.0$ ). Data represent mean \pm s.e.m. ${ }^{*} P<0.05,{ }^{* *} P<0.01$.

agree with survivin protein levels determined by immunohistochemical analysis (Figures 1a and b). As lung epithelial cells, especially bronchial epithelial cells in small airways, have an important function in defending the lung parenchyma against apoptotic stimuli such as physical oxidants, dusts, and infectious insults, it is tempting to speculate that cytoplasmic survivin in bronchial epithelial cells contributes to a constitutive antiapoptotic activity that would benefit the integrity and physiological functioning of the lung.

We found clear evidence of survivin-positive epithelial cells of bronchioles and alveoli in bleomycin-injured lungs (Figures $1 \mathrm{c}-\mathrm{h}$, Figures $2 \mathrm{a}-\mathrm{j}$ and Figures $3 \mathrm{a}-\mathrm{d}$ ), with marked upregulation of survivin mRNA, as determined by RT-qPCR, compared with control lungs (Figure 3e). These findings are consistent with reports of elevated survivin expression in regenerating liver cells after partial hepatectomy, intraperitoneal carbon tetrachloride injection, ${ }^{12}$ and injection of Fas agonistic antibody Jo2 $2^{17}$ in mice and in acinar and ductal cells in rats after induced acute pancreatitis. ${ }^{25}$ Survivin expression was nearly absent or low in controls before treatment, as in normal adult differentiated tissue. Thus, as we expected, survivin may not only function in cancerous tissue cells but also function in epithelial cells of the lung, as in the liver and pancreas, during injury.
The dual role of survivin has been proposed to be associated with different cell compartments. Nuclear, cytoplasmic, and mitochondrial pools of survivin were previously described. ${ }^{2,3,26,27}$ Nuclear survivin facilitates cell cycle progression by inducing cells to exit $G_{1}$ and enter the $S$ phase. ${ }^{28}$ Mitochondrial survivin demonstrates increased stability, which may increase protection from apoptosis. Mitochondrial survivin confers resistance to apoptosis by inhibiting activation of effector caspases. ${ }^{2,3}$ An extracellular pool of survivin retained both antiapoptotic and proliferative activities. Survivin release in the extracellular space was mediated by exosomes and induced by stress-related stimuli. ${ }^{29-31}$

We found survivin-positive results especially in nuclei of epithelial cells of bronchioles and alveoli in day 3 and day 7 models of bleomycin-injured lung (Figures $1 \mathrm{c}-\mathrm{f}$ ), with double-positive results for PCNA (Figures $4 \mathrm{a}, \mathrm{b}, \mathrm{g}-\mathrm{i}$ ). As nuclear survivin would be linked to an ability to regulate cell division, ${ }^{29}$ our data suggest that survivin expression in nuclei with PCNA-positive findings at the early phase of the lung injury reaction in this model may be involved in lung regeneration and proliferation after acute lung injury.

We also found survivin-positive results in the cytoplasm of epithelial cells of bronchioles and alveoli, mainly in day 7 and day 14 models (Figures $1 \mathrm{e}-\mathrm{h}$ and Figures $2 \mathrm{a}-\mathrm{i}$, arrows), with 
a
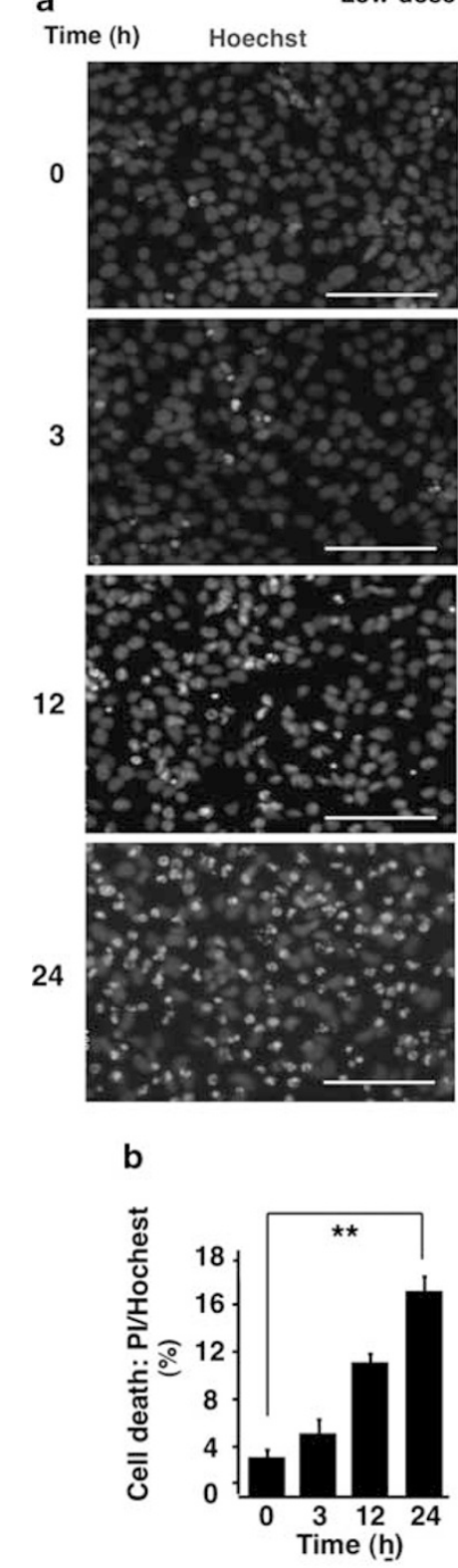

Low-dose bleomycin
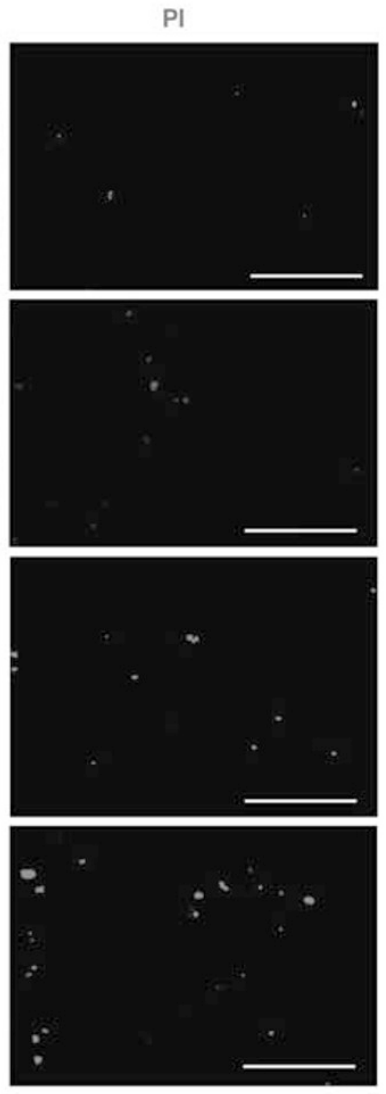

c

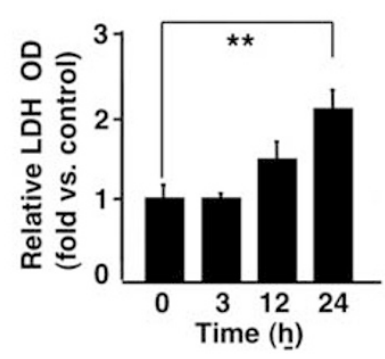

d

Time (h) Hoechst

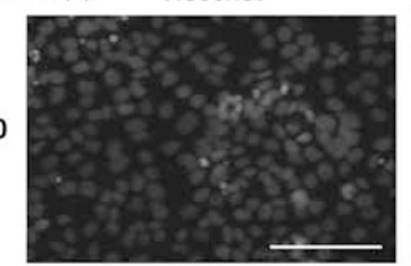

3

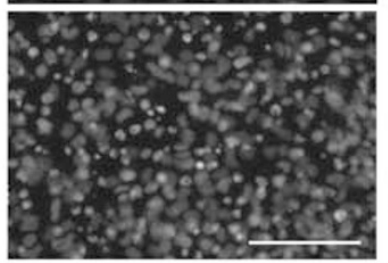

12
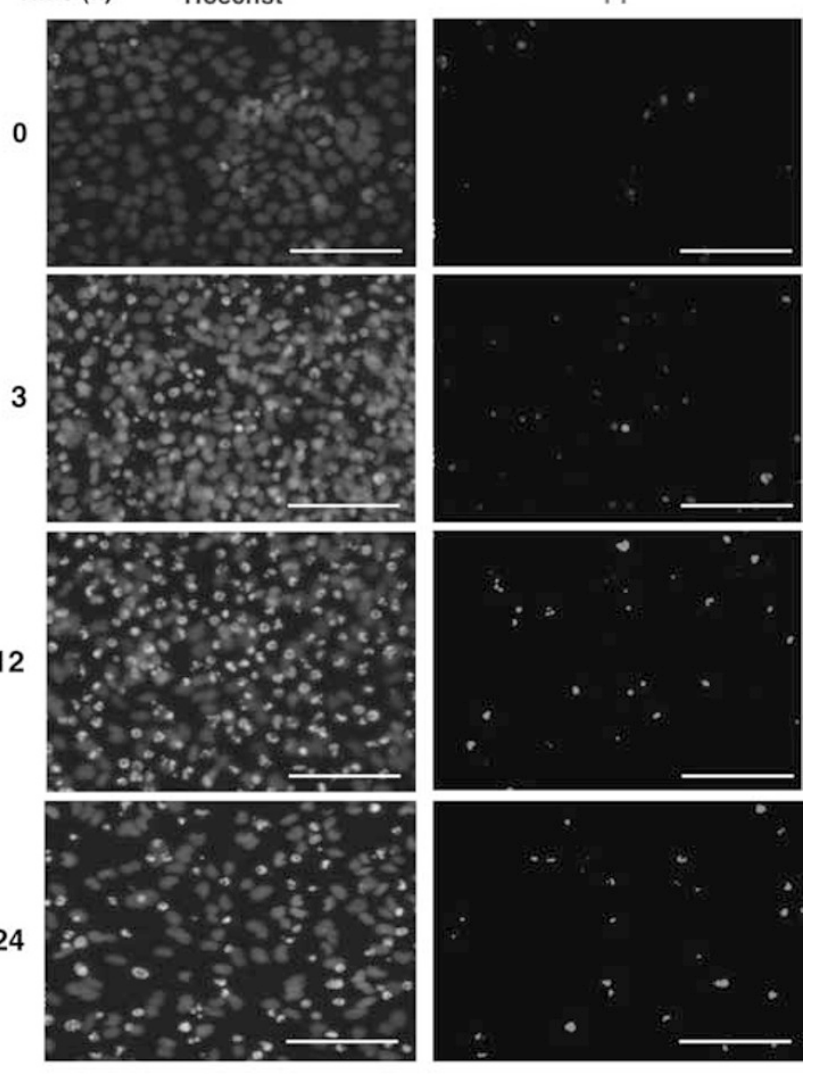

e

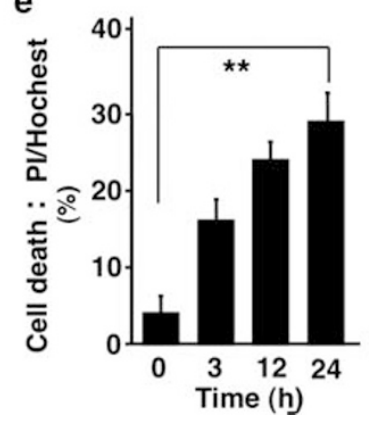

f

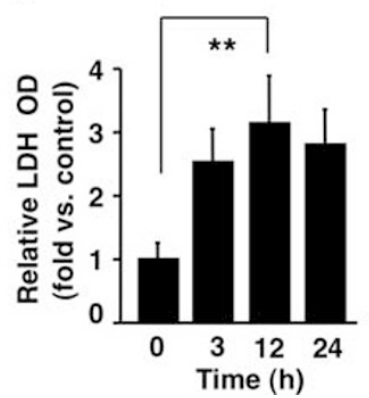

Figure 7 Level of damage of human bronchial cells after bleomycin treatment. (a-d) Images of BEAS-2B cells stained with propidium iodide (PI; dead cells: bright spots) at indicated times after treatment with low-dose bleomycin (a) or high-dose bleomycin (d). Hoechst staining shows nuclei of dead and living cells. Scale bar: $100 \mu \mathrm{m}$. (b, c, e, f) Quantification of cell damage and cell survival after the treatment described in panel a or d, shown as PI/ Hoechst-positive cells (b-e), obtained by manual counting, and relative lactate dehydrogenase (LDH) activity $(\mathbf{c}-\mathbf{f})(n=5)$. Data represent mean \pm s.e.m. ${ }^{* *} P<0.01$.

Smac/DIABLO-positive results (Figures $4 \mathrm{c}$ and $\mathrm{d}$ ) and TUNEL-negative results (Figures $4 \mathrm{e}$ and $\mathrm{f}$, and $\mathrm{j}-\mathrm{l}$ ). In addition, we found survivin-positive intracytoplasmic granules with mitochondria-like structures in the bleomycin-injured lung in the day 14 model (Figure 2j). These findings are consistent with other results of survivin-positive cytoplasm of hepatocytes in Jo2-induced liver injury in mice ${ }^{17}$ and of acinar and ductal cells in $4 \%$ sodium taurocholate-induced acute pancreatitis in rats. ${ }^{25}$ As one mechanism of the antiapoptotic effects of survivin is binding to mitochondrial Smac/DIABLO, ${ }^{4}$ and cytosolic survivin, including mito- chondrial and exosomal survivin, has an essential function in protecting cells from apoptotic stimuli, upregulated survivin with Smac/DIABLO in the cytoplasm of epithelial cells in our mouse model may signify cytoprotection from injury.

These results, together with findings of survivin-positive nuclei and cytoplasm of epithelial cells in human lung DAD lesions (Figures $5 \mathrm{c}-\mathrm{g}$ ), suggest that survivin in epithelial cells may be involved in the pathophysiology of acute lung injury, including apoptosis and regeneration pathways, in mice and humans, which is consistent with our hypothesis. 
a

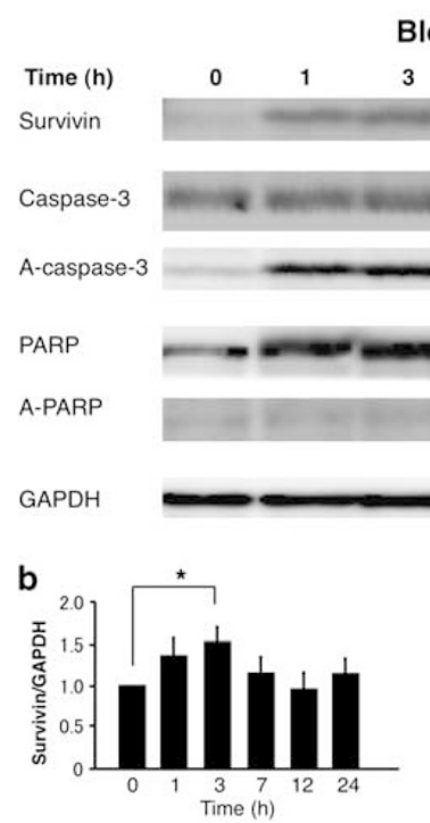

Bleomycin
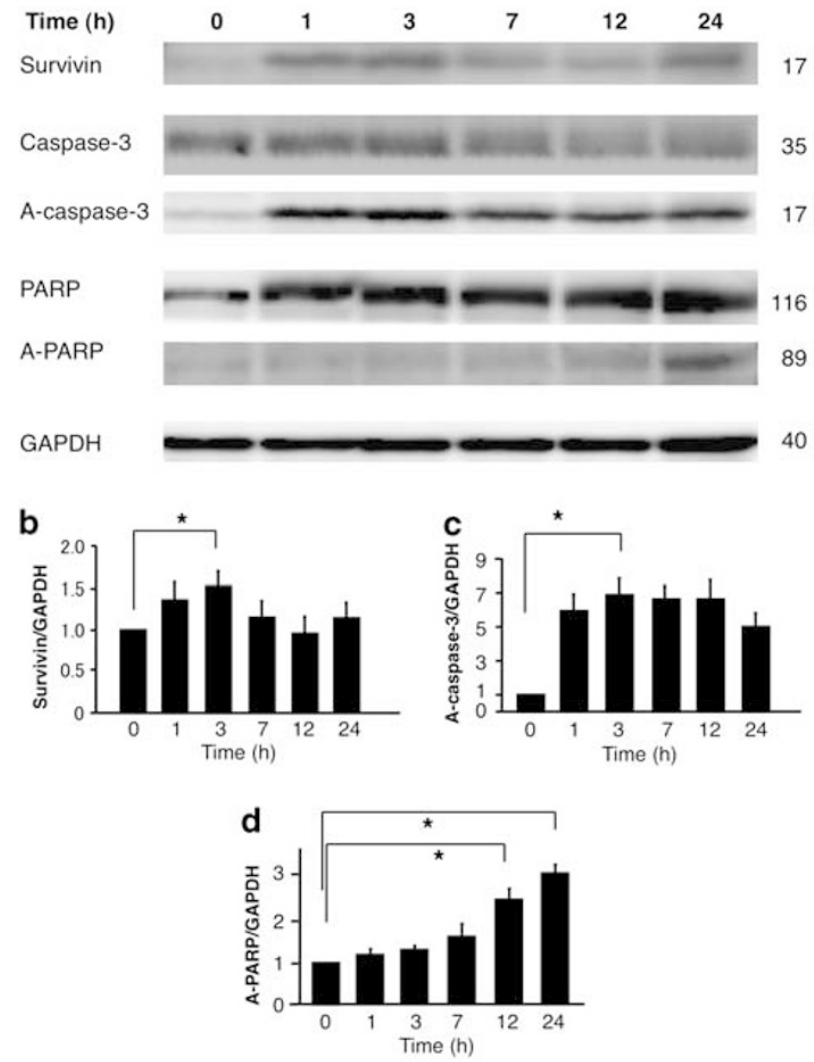

e

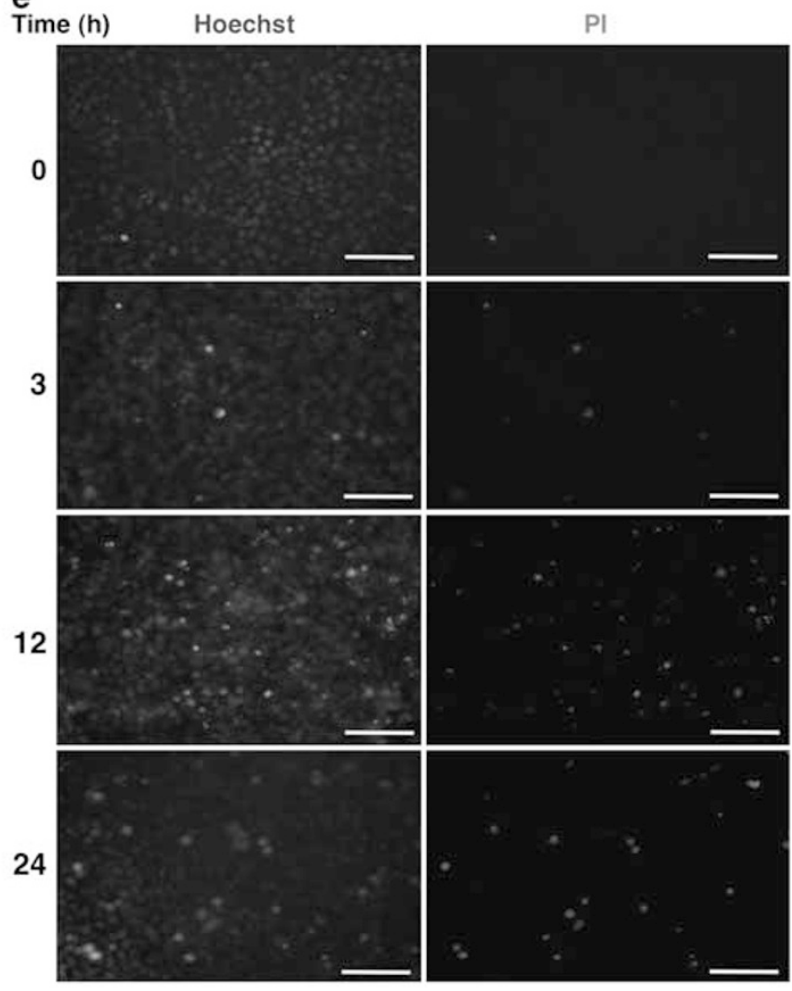

f

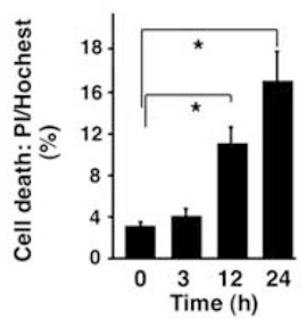

g

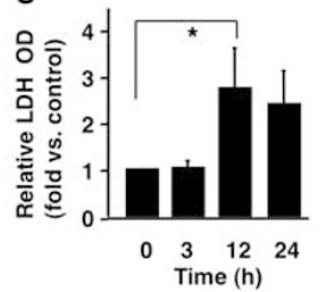

Figure 8 Expression of survivin and level of cell damage in human alveolar cells after bleomycin treatment. (a) A549 cell extracts, at indicated times after treatment with high-dose bleomycin, were subjected to sodium dodecyl sulfate-polyacrylamide gel electrophoresis and were immunoblotted with antibodies against survivin, caspase-3, activated caspase-3 (A-caspase-3), poly (ADP-ribose) polymerase (PARP), activated PARP (A-PARP), and GAPDH (control). (b-d) In five experiments similar to that whose results are shown in panel $\mathbf{a}$, the amounts of each protein were quantified by means of densitometry and were expressed relative to the amount of GAPDH in those samples. Results are reported relative to those of five controls in five experiments (no treatment $=1.0$ ). Data represent mean \pm s.e.m. $(\mathbf{e})$ Images of A549 cells stained with propidium iodide (Pl; dead cells: bright spots) at indicated times after treatment with bleomycin. Hoechst staining shows nuclei of dead and living cells. Scale bar: $100 \mu \mathrm{m}$. (f and $\mathbf{g})$ Quantification of cell damage and cell survival after the treatment described in panel $\mathbf{e}$, shown as $\mathrm{PI} /$ Hoechst-positive cells (f), obtained by manual counting, and relative lactate dehydrogenase $(\mathrm{LDH})$ activity $(\mathbf{g})(n=5)$. Data represent mean \pm s.e.m. ${ }^{*} P<0.05$.

The gene encoding for survivin, BIRC5, generates five major isoforms of the transcript: wild-type survivin, survivin- $\Delta \mathrm{Ex} 3$, survivin- $3 \mathrm{~B}$, survivin- $2 \mathrm{~B}$, and survivin- $2 \alpha$. The first three appear to be cytoprotective, whereas the last two promote apoptosis induction. Some isoforms are also involved in controlling cell division. ${ }^{30-32}$ Our antibody for survivin in vivo did not distinguish these isoforms; therefore, to examine the role of survivin, especially its cytoprotective effect in bleomycin-induced acute injury, and the expression of related molecules, we used cultured lung epithelial BEAS2B and A549 cells and evaluated the cytoprotective effect after suppression or overexpression of survivin in vitro.
We confirmed that survivin was upregulated in bleomycininjured lung epithelial cells in vitro, as well as in vivo, and that bleomycin-induced lung epithelial cell damage in vitro via an apoptotic process involving activation of caspase-3, caspase-7, and PARP (Figures 6a-j and Figures 8a-d). Also, PI-positive cells and LDH activity indicated increased cell damage (Figures $7 \mathrm{a}-\mathrm{f}$ and Figures $8 \mathrm{e}-\mathrm{g}$ ). Then, in this in vitro system, we evaluated the antiapoptotic effect of survivin and found that siRNA-induced survivin suppression rendered lung epithelial cells susceptible to bleomycin-induced damage, with markedly upregulated activation of caspase- 3 , caspase-7, and PARP (Figures $9 \mathrm{a}-\mathrm{e}$ and Figures 10a-c), and also 


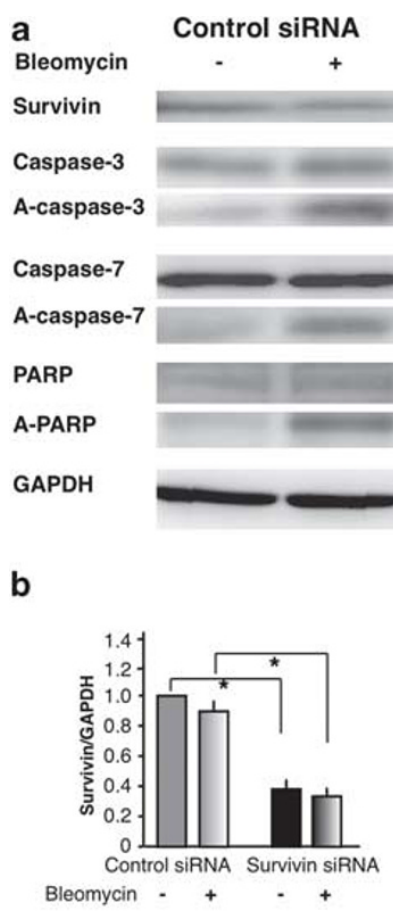

d

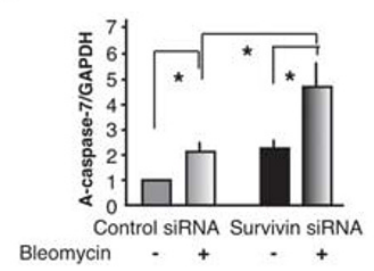

C

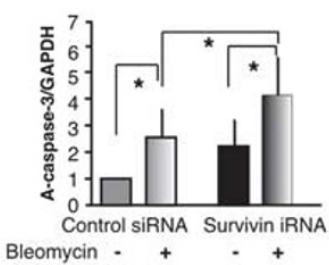

e

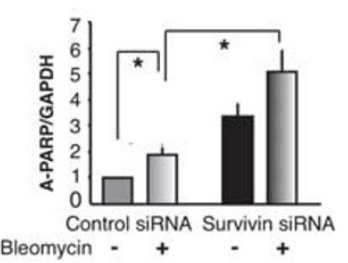

f

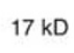

$35 \mathrm{kD}$

$17 \mathrm{kD}$

$35 \mathrm{kD}$

$20 \mathrm{kD}$

$116 \mathrm{kD}$

$89 \mathrm{kD}$

$40 \mathrm{kD}$

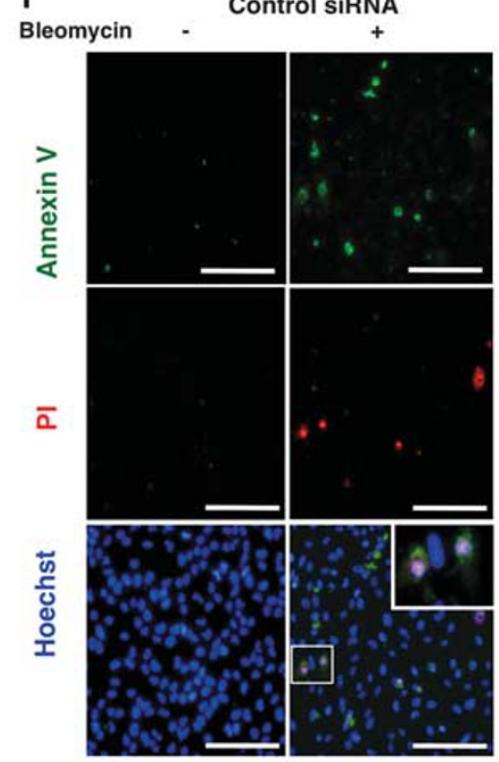

g

Annexin V/Hochest
PV/Hochest

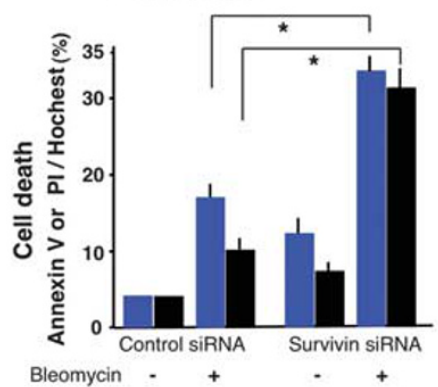

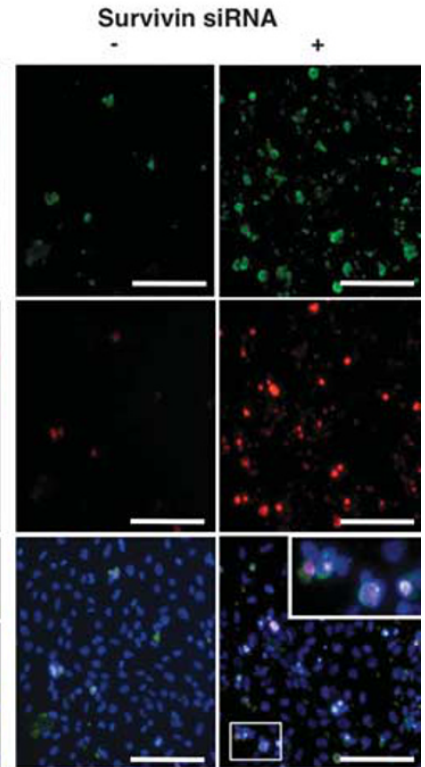

h

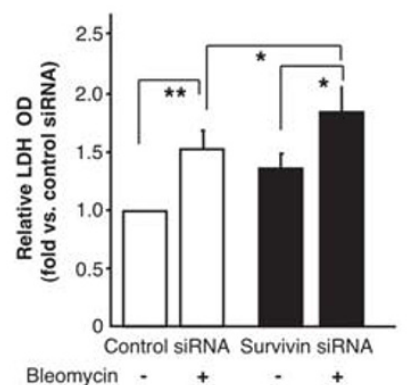

Figure 9 Effect of survivin suppression on bleomycin-induced damage of human bronchial epithelial cells. (a) small interfering RNA (siRNA)-treated cell extracts at $12 \mathrm{~h}$ after low dose bleomycin treatment were subjected to sodium dodecyl sulfate-polyacrylamide gel electrophoresis and were immunoblotted with antibodies against survivin, caspase-3, activated caspase-3 (A-caspase-3), caspase-7, activated caspase-7 (A-caspase-7), poly (ADPribose) polymerase (PARP), activated PARP (A-PARP), and GAPDH (control). (b-e) In five experiments similar to that whose results are shown in panel a, the amounts of each protein were quantified using densitometry and were expressed relative to the amount of GAPDH in those samples. Results are reported relative to those of five controls in five experiments (no treatment $=1.0$ ). Data represent mean \pm s.e.m. $(\mathbf{f}$ ) Images of BEAS-2B cells stained with Annexin V (apoptotic cells: green) or propidium iodide (Pl; dead cells: red) after being treated with survivin siRNA or mock siRNA after $12 \mathrm{~h}$ of bleomycin treatment. Hoechst staining (blue) shows nuclei of dead and living cells. Insets show high-magnification views of areas in rectangles. Scale bar: $100 \mu \mathrm{m}$. (g, h) Quantification of cell damage and cell survival after the treatment described in panel f, shown as Annexin V/Hoechst-positive or PI/ Hoechst-positive cells $(\mathbf{g})$, obtained by manual counting, and relative lactate dehydrogenase (LDH) activity $(\mathbf{h})(n=5)$. Data represent mean \pm s.e.m. ${ }^{*} P<0.05,{ }^{* *} P<0.01$.

increased the number of apoptotic or dead cells and $\mathrm{LDH}$ activity (Figures $9 \mathrm{f}-\mathrm{h}$ and Figures $10 \mathrm{~g}-\mathrm{i}$ ). These results agree with other reports demonstrating that suppression of survivin expression in noncancerous tissues or cells leads to greater sensitivity to apoptotic damage, with evidence from these reports including siRNA targeting in ischemia/ reperfusion-induced rat cardiomyocyte injury ${ }^{33}$ and in staurosporine-induced human endometriotic stromal cell injury $^{34}$ as well as transgenic inactivation in mice, with or without survivin, in Fas ligand-induced liver injury ${ }^{17}$ and folic acid-induced kidney injury. ${ }^{35}$ We also determined that overexpression of survivin by transfection conversely rendered lung epithelial cells resistant to bleomycin-induced damage, with reduced levels of activation of caspase-3, caspase-7, and PARP (Figures 10d-f and Figures 11a-e) and with lower numbers of apoptotic or dead cells and $\mathrm{LDH}$ activity (Figures $10 \mathrm{~h}-\mathrm{j}$ and Figures $11 \mathrm{f}-\mathrm{h}$ ). These results are consistent with reports that survivin overexpression in rat cardiomyocytes led to greater resistance to apoptotic damage in ischemia/reperfusion injury. ${ }^{33}$

These results thus indicate that survivin, acting at the epithelial cell level by a mechanism that depends, in part, on caspase-dependent apoptosis inhibition, is a primary mediator of cytoprotection in acute lung injury.

Recent studies of the molecular dissection of genes associated with the abnormal proliferation of cancer cells 
a

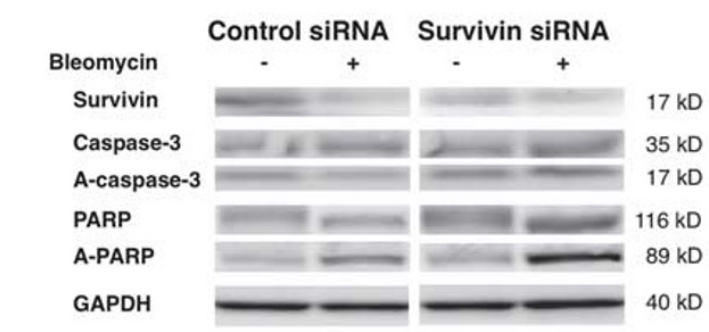

b
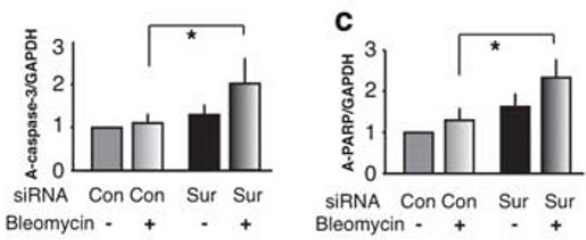

d

Overexpression Control Survivin Control Survivin

Bleomycin

Survivin

Caspase-3

A-caspase-3

PARP

A-PARP

GAPDH
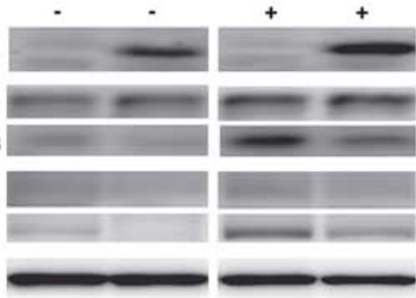

$17 \mathrm{kD}$

$35 \mathrm{kD}$

$17 \mathrm{kD}$

$116 \mathrm{kD}$

$89 \mathrm{kD}$

$40 \mathrm{kD}$

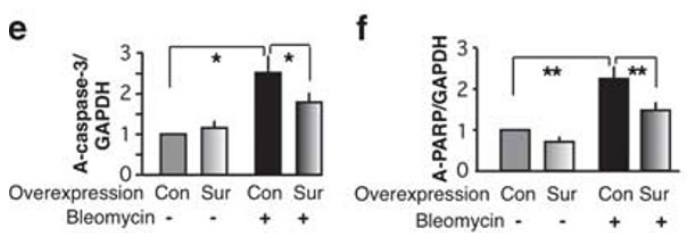

g
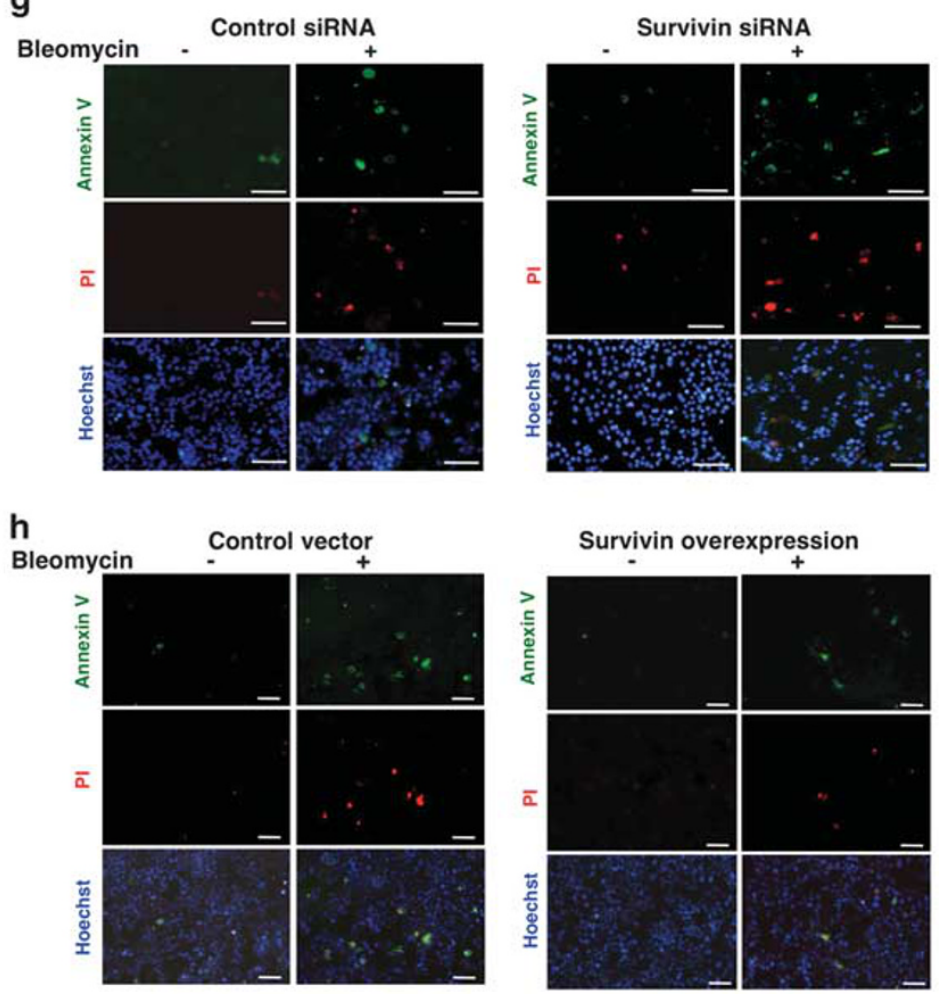

i

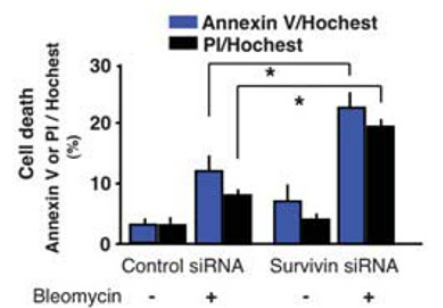

Figure 10 Effect of survivin suppression or overexpression on bleomycin-induced damage of human alveolar epithelial cells. (a) small interfering RNA (siRNA)-treated A549 cell extracts at $12 \mathrm{~h}$ after bleomycin treatment were subjected to sodium dodecyl sulfate-polyacrylamide gel electrophoresis (SDSPAGE) and were immunoblotted with antibodies against survivin, caspase-3, activated caspase-3 (A-caspase-3), poly(ADP-ribose) polymerase (PARP), activated PARP (A-PARP), and GAPDH (control). (b and $\mathbf{c}$ ) In five experiments similar to that whose results are shown in panel a, the amounts of A-caspase-3 and A-PARP were quantified using densitometry and were expressed relative to the amount of GAPDH in those samples. Results are reported relative to those of five controls in five experiments (no treatment $=1.0$ ). Data represent mean \pm s.e.m. Con, control; Sur, survivin.

(d) Transfected cell extracts at $12 \mathrm{~h}$ after bleomycin treatment were subjected to SDS-PAGE and were immunoblotted with antibodies against survivin, caspase-3, A-caspase-3, PARP, A-PARP, and GAPDH (control). (e, f) In five experiments similar to that whose results are shown in panel d, the amounts of A-caspase-3 and A-PARP were quantified using densitometry and were expressed relative to the amount of GAPDH in those samples. Results are reported relative to those of five controls in five experiments (no treatment $=1.0$ ). Data represent mean \pm s.e.m. (g) Images of A549 cells stained with Annexin V (green) or propidium iodide (Pl; red) after being treated with survivin siRNA or mock siRNA after $12 \mathrm{~h}$ of bleomycin treatment. Hoechst staining (blue) shows nuclei of dead and living cells. Scale bar: $100 \mu \mathrm{m}$. (h) Images of A549 cells stained with Annexin V or PI after being treated with survivin transfection or mock transfection after $12 \mathrm{~h}$ of bleomycin treatment. Hoechst staining (blue) shows nuclei of dead and living cells. Scale bar: $50 \mu \mathrm{m}$. (i and $\mathbf{j}$ ) Quantification of cell damage and cell survival after the treatment described in panels $\mathbf{g}$ and $\mathbf{h}$, respectively, shown as Annexin V/ Hoechst-positive or PI/Hoechst-positive cells obtained by manual counting $(n=5)$. Data represent mean \pm s.e.m. ${ }^{*} P<0.05,{ }^{* *} P<0.01$.

identified survivin as a possible gene responsible for progression of cancer and as a potential molecular therapeutic target for these pathological conditions. ${ }^{8}$ Survivin may also have physiological functions in regulating proliferation and survival in normal tissues, as shown by our data and other reports; ${ }^{8,10}$ however, its expression in normal tissues is significantly lower than that in cancer cells. The fact that the constitutively high survivin expression in cancer cells is not under the same regulatory control as survivin expression in noncancerous cells should be noted. For example, the DNA-protein interaction in the survivin promoter differed for nuclear proteins isolated from normal and cancer cells, ${ }^{36}$ 
a

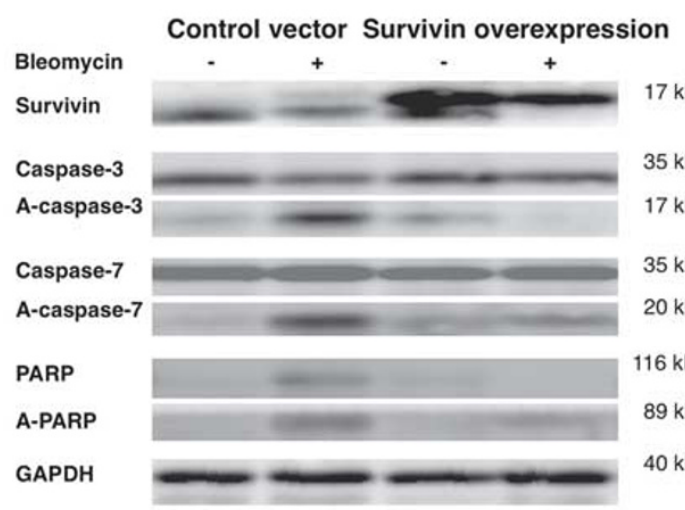

b
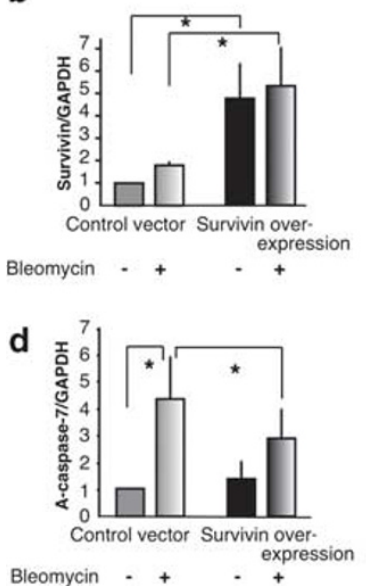

C
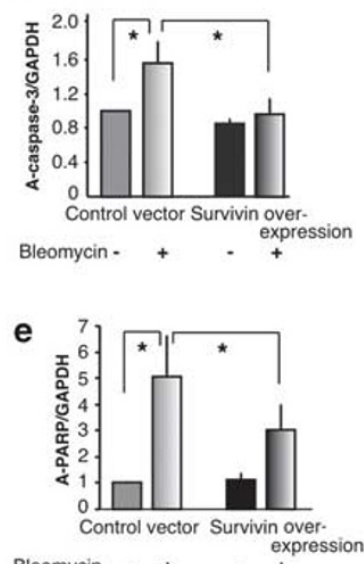

f

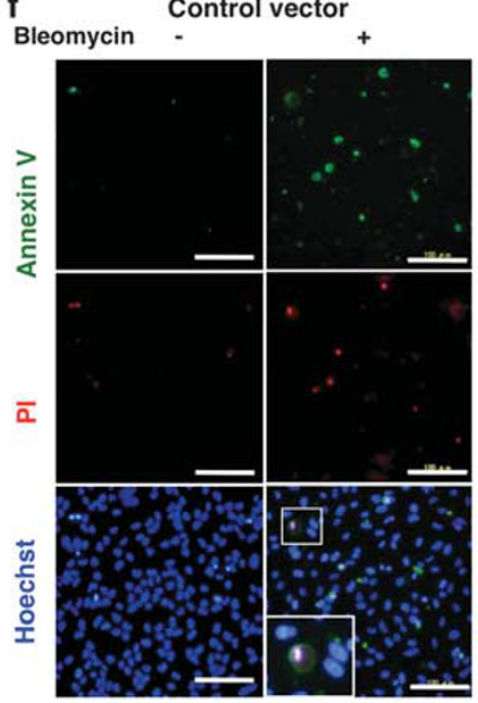

g Annexin V/Hochest - PUHochest

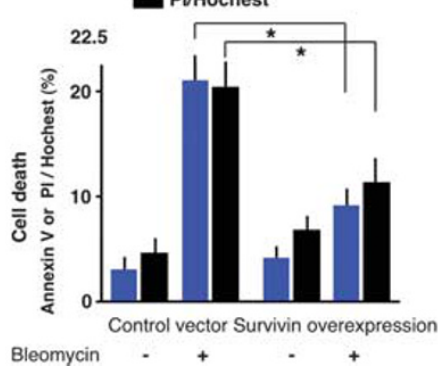

Survivin overexpression

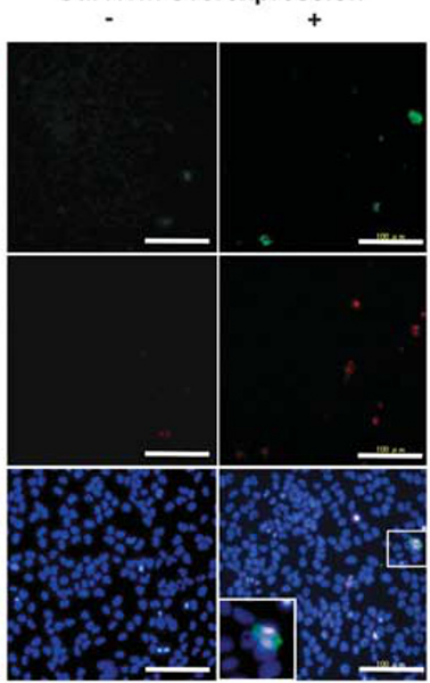

h

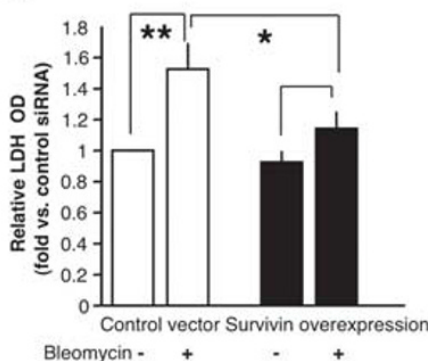

Figure 11 Effect of survivin overexpression on bleomycin-induced damage of human bronchial epithelial cells. (a) Transfected cell extracts at $12 \mathrm{~h}$ after bleomycin treatment were subjected to sodium dodecyl sulfate-polyacrylamide gel electrophoresis and were immunoblotted with antibodies against survivin, caspase-3, activated caspase-3 (A-caspase-3), caspase-7, activated caspase-7 (A-caspase-7), poly (ADP-ribose) polymerase (PARP), activated PARP (A-PARP), and GAPDH (control). (b-e) In five experiments similar to that whose results are shown in panel a, the amounts of each protein were quantified using densitometry and were expressed relative to the amount of GAPDH in those samples. Results are reported relative to those of five controls in five experiments (no treatment $=1.0$ ). Data represent mean \pm s.e.m. (f) Images of BEAS-2B cells stained with Annexin V (apoptotic cells: green) or propidium iodide (PI; dead cells: red) after being treated with survivin transfection or mock transfection after $12 \mathrm{~h}$ of bleomycin treatment. Hoechst staining (blue) shows nuclei of dead and living cells. Scale bar: $100 \mu \mathrm{m}$. (g, h) Quantification of cell damage and cell survival after the treatment described in panel f, shown as Annexin V/Hoechst-positive or PI/Hoechst-positive cells (g), obtained by manual counting, and relative lactate dehydrogenase $(\mathrm{LDH})$ activity $(\mathbf{h})(n=5)$. Data represent mean \pm s.e.m. ${ }^{*} P<0.05,{ }^{* *} P<0.01$.

and a mutation in the survivin promoter correlated with survivin mRNA overexpression in cancer cells. ${ }^{37}$ However, reports also noted that survivin regulated apoptosis and proliferation of normal cells and cancer cells using some of the same pathways. For example, not only in normal hematopoietic cells but also in hepatoma cells, survivin regulated apoptosis through p21-dependent pathways. ${ }^{38}$ Continued investigations of the mechanisms of regulating survivin expression and function in normal cells and cancer cells will help identify critical differences in survivin activity that can aid development of novel strategies utilizing survivin functions.

In conclusion, this study provides evidence that survivin, acting not only at cancerous cell level but also at the epithelial cell level in mice and humans, is an essential mediator of cytoprotection in lung injury. As many human tumors manifest high survivin expression, using survivin as a target for cancer therapeutic agents, has been investigated. ${ }^{10}$ However, interstitial lung disease associated with use of such drugs has developed, ${ }^{39}$ and whether using survivin as a target in cancer treatment would be toxic to normal human lung cells and tissues has not been clarified. ${ }^{10}$ Understanding the precise role of survivin in normal lung cells and tissues as well as in cancer cells and tissues is required for the therapeutic development of survivin.

Supplementary Information accompanies the paper on the Laboratory Investigation website (http://www.laboratoryinvestigation.org) 


\section{ACKNOWLEDGEMENTS}

We thank Ms Judith B. Gandy for her excellent editing of the manuscript. This study was supported by grants-in-aid for scientific research from the Ministry of Education, Culture, Sports, Science and Technology (MEXT) of Japan. This study was also partly supported by a grant to the Diffuse Lung Diseases Research Group from the Ministry of Health, Labor and Welfare of Japan.

\section{DISCLOSURE/CONFLICT OF INTEREST}

The authors declare no conflict of interest.

1. Song Z, Yao X, Wu M. Direct interaction between survivin and Smac/ DIABLO is essential for the anti-apoptotic activity of survivin during taxol-induced apoptosis. J Biol Chem 2003;278:23130-23140.

2. Dohi $T$, Beltrami E, Wall NR, et al. Mitochondrial survivin inhibits apoptosis and promotes tumorigenesis. J Clin Invest 2004;114: 1117-1127.

3. Dohi T, Okada K, Xia F, et al. An IAP-IAP complex inhibits apoptosis. J Biol Chem 2004;279:34087-34090.

4. Ceballos-Cancino G, Espinosa M, Maldonado V, et al. Regulation of mitochondrial Smac/DIABLO-selective release by survivin. Oncogene 2007;26:7569-7575.

5. Marusawa $\mathrm{H}$, Matsuzawa $\mathrm{S}$, Welsh $\mathrm{K}$, et al. HBXIP functions as a cofactor of survivin in apoptosis suppression. EMBO J 2003;22:2729-2740.

6. Li F, Ambrosini G, Chu EY, et al. Control of apoptosis and mitotic spindle checkpoint by survivin. Nature 1998;396:580-584.

7. Dallaglio K, Marconi A, Pincelli C. Survivin: a dual player in healthy and diseased skin. J Invest Dermatol 2012;132:18-27.

8. O'Driscoll L, Linehan R, Clynes M. Survivin: role in normal cells and in pathological conditions. Curr Cancer Drug Targets 2003;3:131-152.

9. Uren $A G$, Wong $L$, Pakusch $M$, et al. Survivin and the inner centromere protein INCENP show similar cell-cycle localization and gene knockout phenotype. Curr Biol 2000;10:1319-1328.

10. Li F, Brattain MG. Role of the Survivin gene in pathophysiology. Am J Pathol 2006;169:1-11.

11. Chiou SK, Moon WS, Jones MK, et al. Survivin expression in the stomach: implications for mucosal integrity and protection. Biochem Biophys Res Commun 2003;305:374-379.

12. Deguchi $M$, Shiraki $K$, Inoue $H$, et al. Expression of survivin during liver regeneration. Biochem Biophys Res Commun 2002;297:59-64.

13. Conway EM, Zwerts F, Van Eygen V, et al. Survivin-dependent angiogenesis in ischemic brain: molecular mechanisms of hypoxiainduced up-regulation. Am J Pathol 2003;163:935-946.

14. Jones MK, Padilla OR, Webb NA, et al. The anti-apoptosis protein survivin, mediates gastric epithelial cell cytoprotection against ethanol-induced injury via activation of the p34(cdc2) cyclindependent kinase. J Cell Physiol 2008;215:750-764.

15. Dohi T, Altieri DC. Mitochondrial dynamics of survivin and "four dimensional" control of tumor cell apoptosis. Cell Cycle 2005:4:21-23.

16. Chen J, Wu W, Tahir SK, et al. Down-regulation of survivin by antisense oligonucleotides increases apoptosis, inhibits cytokinesis and anchorage-independent growth. Neoplasia 2000;2:235-241.

17. Conway EM, Pollefeyt S, Steiner-Mosonyi M, et al. Deficiency of survivin in transgenic mice exacerbates Fas-induced apoptosis via mitochondrial pathways. Gastroenterology 2002;123:619-631.

18. Rossi AG, Sawatzky DA, Walker A, et al. Cyclin-dependent kinase inhibitors enhance the resolution of inflammation by promoting inflammatory cell apoptosis. Nat Med 2006;12:1056-1064.
19. Mura M, Binnie M, Han B, et al. Functions of type II pneumocytederived vascular endothelial growth factor in alveolar structure, acute inflammation, and vascular permeability. Am J Pathol 2010;176: $1725-1734$.

20. Ikeda KST, Tate G, Mitsuya T. Multiple immunoenzyme labeling using heat treatment combined with the polymer method: an analysis of the appropriate inactivation conditions of primary antibodies. Acta Histochem 2011;113:117-124.

21. Fukuda S, Pelus LM. Survivin, a cancer target with an emerging role in normal adult tissues. Mol Cancer Ther 2006;5:1087-1098.

22. Monzo $M$, Rosell $R$, Felip $E$, et al. A novel anti-apoptosis gene: re-expression of survivin messenger RNA as a prognosis marker in non-small-cell lung cancers. J Clin Oncol 1999;17:2100-2104.

23. Jin $Q$, Menter DG, Mao $L$, et al. Survivin expression in normal human bronchial epithelial cells: an early and critical step in tumorigenesis induced by tobacco exposure. Carcinogenesis 2008;29:1614-1622.

24. Jones MK, Padilla OR, Zhu E. Survivin is a key factor in the differential susceptibility of gastric endothelial and epithelial cells to alcoholinduced injury. J Physiol Pharmacol 2010;61:253-264.

25. Tashiro $M$, Nakamura $H$, Taguchi $M$, et al. Expression of survivin after acute necrohemorrhagic pancreatitis in rats. Pancreas 2003;26:160-165.

26. Fortugno $\mathrm{P}$, Wall $\mathrm{NR}$, Giodini $\mathrm{A}$, et al. Survivin exists in immunochemically distinct subcellular pools and is involved in spindle microtubule function. J Cell Sci 2002;115:575-585.

27. Colnaghi R, Connell CM, Barrett RM, et al. Separating the antiapoptotic and mitotic roles of survivin. J Biol Chem 2006;281: 33450-33456.

28. Connell CM, Wheatley SP, McNeish IA. Nuclear survivin abrogates multiple cell cycle checkpoints and enhances viral oncolysis. Cancer Res 2008;68:7923-7931.

29. Srinivasula SM, Ashwell JD. IAPs: what's in a name? Mol Cell 2008;30:123-135.

30. Mahotka $C$, Wenzel $M$, Springer $E$, et al. Survivin- $\Delta E \times 3$ and survivin-2B two novel splice variants of the apoptosis inhibitor survivin with different antiapoptotic properties. Cancer Res 1999;59:6097-6102.

31. Caldas H, Jiang Y, Holloway MP, et al. Survivin splice variants regulate the balance between proliferation and cell death. Oncogene 2005;24:1994-2007.

32. Knauer SK, Bier C, Schlag $P$, et al. The survivin isoform survivin-3B is cytoprotective and can function as a chromosomal passenger complex protein. Cell Cycle 2007;6:1502-1509.

33. Si $R$, Tao $L$, Zhang HF, et al. Survivin: a novel player in insulin cardioprotection against myocardial ischemia/reperfusion injury. J Mol Cell Cardiol 2011;50:16-24.

34. Watanabe A, Taniguchi $F$, Izawa $M$, et al. The role of survivin in the resistance of endometriotic stromal cells to drug-induced apoptosis. Hum Reprod 2009;24:3172-3179.

35. Kindt N, Menzebach A, Van de Wouwer M, et al. Protective role of the inhibitor of apoptosis protein, survivin, in toxin-induced acute renal failure. FASEB J 2008;22:510-521.

36. Li F. Survivin study: what is the next wave? J Cell Physiol 2003;197:8-29.

37. $\mathrm{Xu} \mathrm{Y}$, Fang $F$, Ludewig $G$, et al. A mutation found in the promote region of the human survivin gene is correlated to overexpression of survivin in cancer cells. DNA Cell Biol 2004;23:527-537.

38. Fukuda S, Mantel CR, Pelus LM. Survivin regulates hematopoietic progenitor cell proliferation through p21WAF1/Cip1-dependent and -independent pathways. Blood 2004;103:120-127.

39. Min JH, Lee HY, Lim H, et al. Drug-induced interstitial lung disease in tyrosine kinase inhibitor therapy for non-small cell lung cancer: a review on current insight. Cancer Chemother Pharmacol 2011;68: 1099-1109. 